\title{
Optimal lifetime consumption and investment under drawdown constraint
}

\author{
Romuald Elie* $\quad$ Nizar Touzi ${ }^{\dagger}$
}

November 28, 2007

First version: October 21, 2006

\begin{abstract}
We consider the infinite horizon optimal consumption-investment problem under the drawdown constraint, i.e. the wealth process never falls below a fixed fraction of its running maximum. We assume that the risky asset is driven by the constant coefficients Black and Scholes model. For a general class of utility functions, we provide the value function in explicit form, and we derive closed-form expressions for the optimal consumption and investment strategy.
\end{abstract}

Key words: portfolio allocation, drawdown constraint, duality, verification.

AMS 2000 subject classifications: 91B28, 35K55, 60H30.

\section{Introduction}

Since the seminal papers of Merton [16, 17], there has been an extensive literature on the problem of optimal consumption and investment decision in financial markets subject to imperfections. The case of incomplete markets was first considered by Cox and Huang [4] and Karatzas, Lehoczky and Shreve [13]. Cvitanić and Karatzas [5] considered the case where the agent portfolio is restricted to take values in some given closed convex set. He and Pagès [10] and El Karoui and Jeanblanc [8] extended the Merton model to allow for the presence of labor income. Constantinides and Magill [3], Davis and Norman [7], and Shreve and Soner [21] considered the case where the risky asset is subject to proportional transaction costs. Ben Tahar, Soner and Touzi [2] considered the case where the sales of the risky asset are subject to taxes on the capital gains.

In this paper, we study the infinite horizon optimal consumption and investment problem when the wealth never falls below a fixed fraction of its current maximum. This is the so-called drawdown constraint. Fund managers do offer this type of guarantee in order to satisfy the aversion to deception of the investors.

\footnotetext{
${ }^{*}$ CEREMADE \& CREST, elie@ceremade.dauphine.fr

${ }^{\dagger}$ Centre de Mathématiques Appliquées, Ecole Polytechnique Paris, touzi@cmap.polytechnique.fr.
} 
The drawdown constraint on the wealth accumulation of the fund manager was first considered by Grossman and Zhou [9] for an agent maximizing the long term growth rate of the expected utility of final wealth, with no intermediate consumption. Their main result is that the optimal investment in the risky asset is an explicit constant proportion of the difference between the current wealth and its running maximum. Cvitanic and Karatzas [6] developed a beautiful martingale approach to the Grossman and Zhou [9] problem, with zero interest rate, which makes the analysis much simpler and allows for a more general class of price processes. Nevertheless, Klass and Nowicki [14] show that the strategy proposed in Grossman and Zhou [9] does not retain its optimal long term growth property when generalized to the discrete time setting. A general criticism that one may formulate about the long term growth rate criterion is that it only provides the asymptotic optimal behavior of the fund manager. In other words, there is no penalization for using an arbitrary strategy as long as it coincides with the Grossman and Zhou [9] optimal strategy after some given fixed point in time.

In a recent paper, Roche [19] studied the classical Merton problem, which consists in maximizing the infinite horizon power utility of consumption, for a fund manager subject to the drawdown constraint. The main contribution of [19] is to guess a solution of the dynamic programming equation, and to provide some qualitative properties of the corresponding optimal consumption-investment strategy. The homogeneity of the power utility is the key-property in order to guess the candidate solution. Notice that Roche [19] does not provide any argument to verify that his candidate solution is indeed the value function of the optimal consumption-investment problem.

In this paper, we consider the infinite horizon utility maximization of future consumption, under the drawdown constraint. Our problem has two differences with [19]. First, the utility function is a general $C^{1}$ increasing and strictly concave function whose asymptotic elasticity (see [15]) is bounded by some level depending on the drawdown parameter. Second, following Cvitanić and Karatzas [6], we only address the zero interest rate setting. Notice that, because the discounted value of the maximum wealth is different from the discounted value of the maximum wealth, our setting does not correspond to a simple reduction of the non-zero interest rate setting by usual discounting. We leave this more general context to future work.

Our main result is to derive an explicit expression for the value function of the fund manager, together with the optimal consumption and investment strategy. The key-idea in order to guess the candidate solution is to pass from the dynamic programming equation to the partial differential equation (PDE) satisfied by the dual indirect utility function. The latter PDE being linear inside the state space domain, one can easily account for the Neumann condition related to the drawdown constraint, and derive an explicit candidate solution for any utility function. In order to prove that the thus derived candidate solution is indeed the value function of our optimal consumption-investment problem, we use a verification argument which requires a convenient transversality condition. The verification argument is the main technical step where the above mentioned restrictions on the utility functions are required.

For a general utility function, the qualitative properties of the optimal consumption and 
investment strategies of the problem turn out to be similar to those stated by Roche in the power utility case. In particular, we find a utility-independent level of the drawdown constraint above which the optimal investment in the risky security vanishes when the maximum wealth is reached. In other words, for a large drawdown parameter, the investor avoids to make the drawdown constraint more stringent by holding the current maximum wealth process constant. Below this utility-independent level of the drawdown parameter, the current maximum of the optimal wealth process is not flat.

The paper is organized as follows. Section 2 is devoted to the formulation of the problem. Our solution approach is based on the verfication result reported in Section 3 which suggest to seek for a solution by solving the corresponding dynamic programming equation. A closed-form solution of this equation is obtained in Section 4. The main result of the paper is reported in Section 5 and states that our candidate solution indeed coincides with the value function of the utility maximization problem, and provides the corresponding optimal investment and consumption policy. Section 6 is dedicated to the proof of the main theorem by verifying that the candidate solution satisfies the conditions of the verification result.

\section{Problem formulation}

Throughout this paper, we consider a complete filtered probability space $\left(\Omega, \mathcal{F}, \mathbb{F}=\left\{\mathcal{F}_{t}\right\}_{t \geq 0}, \mathbb{P}\right)$ endowed with a Brownian motion $W=\left\{W_{t}, 0 \leq t \leq T\right\}$ with values in $\mathbb{R}$.

The financial market consists of a non-risky asset, with process normalized to unity, and one risky asset with price process defined by the Black and Scholes model :

$$
d S_{t}=\sigma S_{t}\left(d W_{t}+\lambda d t\right)
$$

where $\sigma>0$ is the volatility parameter, and $\lambda \in \mathbb{R}$ is a constant risk premium.

The normalization of the non-risky asset to unity is as usual a reduction of the model obtained by taking this asset as a numéraire. Hence, all amounts are evaluated in terms of their discounted values.

For any continuous process $\left\{M_{t}, t \geq 0\right\}$, we shall denote by

$$
M_{t}^{*}:=\sup _{0 \leq r \leq t} M_{r}, \quad t \geq 0,
$$

the corresponding running maximum process, and we recall that

$$
M^{*} \text { is non-decreasing and } \int_{0}^{\infty}\left(M_{t}^{*}-M_{t}\right) d M_{t}^{*}=0 .
$$

\subsection{Consumption-portfolio strategies and the drawdown constraint}

We next introduce the set of consumption-investment strategies whose induced wealth process $X$ satisfies the drawdown constraint

$$
X_{t} \geq \alpha X_{t}^{*} \text { for every } t \geq 0, \text { a.s. },
$$

where $\alpha$ is some given parameter in the interval $[0,1)$. 
A portfolio strategy is an $\mathbb{F}$-adapted process $\theta=\left\{\theta_{t}, t \geq 0\right\}$, with values in $\mathbb{R}$, satisfying the integrability condition

$$
\int_{0}^{T}\left|\theta_{t}\right|^{2} d t<\infty \text { a.s. for all } T>0
$$

A consumption strategy is an $\mathbb{F}$-adapted process $C=\left\{C_{t}, t \geq 0\right\}$, with values in $\mathbb{R}_{+}$, satisfying

$$
\int_{0}^{T} C_{t} d t<\infty \text { a.s. for all } T>0
$$

Here, $\theta_{t}$ and $C_{t}$ denote respectively the amount invested in the risky asset and the consumption rate at time $t$. By the self-financing condition, the wealth process induced by such a pair $(C, \theta)$ is defined by

$$
X_{t}^{x, C, \theta}=x-\int_{0}^{t} C_{r} d r+\int_{0}^{t} \sigma \theta_{r}\left(d W_{r}+\lambda d r\right) \quad t \geq 0
$$

where $x$ is some given initial positive capital. We shall denote by $\mathcal{A}_{\alpha}(x)$ the collection of all such consumption-investment strategies whose corresponding wealth process satisfies the drawdown constraint (2.2).

Remark 2.1 The set of admissible strategies $\mathcal{A}_{\alpha}(x)$ is a relaxation of the set of strategies considered by Cvitanić and Karatzas [6] in a no-consumption framework, in the sense that we allow the drawdown constraint to be binding, see the subsequent subsection 2.2. Notice however that our model corresponds to that of Grossman and Zhou [9] only in the zero interest case setting. This is due to the simple fact that the discounted current maximum wealth does not coincide with the maximum of the discounted wealth.

\subsection{Admissible strategies with strict drawdown constraint}

In this subsection, we consider the subset $\mathcal{A}_{\alpha}^{0}\left(X_{0}\right)$ of all strategies $(C, \theta) \in \mathcal{A}_{\alpha}\left(X_{0}\right)$ which satisfy the strict drawdown condition

$$
X_{t}>\alpha X_{t}^{*} \text { for every } t \geq 0 \text {. }
$$

For those strategies, one may define the consumption and the investment decisions in terms of proportions of the difference $X_{t}-\alpha X_{t}^{*}$ :

$$
c_{t}=\frac{C_{t}}{X_{t}-\alpha X_{t}^{*}} \quad \text { and } \quad \pi_{t}=\frac{\theta_{t}}{X_{t}-\alpha X_{t}^{*}} .
$$

By the continuity of the processes $X$ and $X^{*}$, it follows that the pair process $(c, \pi)$ is $\mathbb{F}$-adapted, takes values in $\mathbb{R}_{+} \times \mathbb{R}$ and satisfies

$$
\int_{0}^{T} c_{t} d t+\int_{0}^{T}\left|\pi_{t}\right|^{2} d t<\infty \text { a.s. for every } T>0,
$$


The following argument, adapted from Cvitanic and Karatzas [6], shows that for any $\mathbb{F}$-adapted processes $(c, \pi)$ with values in $\mathbb{R}_{+} \times \mathbb{R}$ satisfying (2.8), the stochastic differential equation

$$
d X_{t}=\left(X_{t}-\alpha X_{t}^{*}\right)\left(\pi_{t} \frac{d S_{t}}{S_{t}}-c_{t} d t\right), \quad t \geq 0
$$

has a unique explicit solution, given by (2.14) below, satisfying the strict drawdown constraint (2.6). Hence the subset $\mathcal{A}_{\alpha}^{0}\left(X_{0}\right)$ can be described by the pair process $(c, \pi)$ satisfying (2.8) without refering to the drawdown constraint, and coincides with the set of consumption-investment strategies considered in [6].

The key ingredient for the construction of a solution to (2.9) is to introduce the process

$$
\tilde{X}_{t}:=\left(X_{t}-\alpha X_{t}^{*}\right)\left(X_{t}^{*}\right)^{\frac{\alpha}{1-\alpha}}, \quad t \geq 0 .
$$

By Itô's Lemma together with (2.1), it follows that

$$
\begin{aligned}
d \tilde{X}_{t} & =\left(X_{t}^{*}\right)^{\frac{\alpha}{1-\alpha}}\left(\frac{\alpha}{1-\alpha}\left(\frac{X_{t}}{X_{t}^{*}}-1\right) d X_{t}^{*}+d X_{t}\right) \\
& =\tilde{X}_{t}\left[\left(\lambda \sigma \pi_{t}-c_{t}\right) d t+\sigma \pi_{t} d W_{t}\right] .
\end{aligned}
$$

Since $(c, \pi)$ satisfies $(2.8)$, the latter linear SDE defines a unique solution given by:

$$
\tilde{X}_{t}=\tilde{X}_{0} \exp \left[\int_{0}^{t}\left(-c_{r}+\lambda \sigma \pi_{r}-\frac{1}{2}\left|\sigma \pi_{r}\right|^{2}\right) d r+\int_{0}^{t} \sigma \pi_{r} d W_{r}\right], t \geq 0,
$$

where

$$
\tilde{X}_{0}=(1-\alpha)\left(X_{0}\right)^{\frac{1}{1-\alpha}}
$$

One also easily checks that

$$
\tilde{X}_{t}^{*}=(1-\alpha)\left(X_{t}^{*}\right)^{\frac{1}{1-\alpha}}
$$

so that the unique solution of (2.9) is obtained by combining (2.10), (2.12) and (2.13):

$$
X=\left(\tilde{X}+\frac{\alpha}{1-\alpha} \tilde{X}^{*}\right)\left(\frac{\tilde{X}^{*}}{1-\alpha}\right)^{-\alpha} \text {. }
$$

Notice that $\tilde{X}$ is strictly positive. Then it follows from (2.10) that the process $X$ defined by (2.14) satisfies the strict drawdown constraint (2.6).

\subsection{The optimal consumption-investment problem}

We now formulate the optimal consumption-investment problem which will be the focus of this paper. Throughout this paper, we consider a utility function

$$
U: \mathbb{R}_{+} \rightarrow \mathbb{R} \quad C^{1} \text {, concave, satisfying } U^{\prime}(0+)=\infty \text { and } U^{\prime}(\infty)=0 \text {. }
$$


For a given initial capital $x>0$, the optimal consumption-investment problem under drawdown constraint is defined by :

$$
u_{0}^{\alpha}:=\sup _{(C, \theta) \in \mathcal{A}_{\alpha}(x)} J(C, \theta) \text { where } J(C, \theta):=\mathbb{E}\left[\int_{0}^{\infty} e^{-\beta t} U\left(C_{t}\right) d t\right],
$$

and $\beta>0$ is the subjective discount factor which expresses the preference of the agent for the present.

For $\alpha=0, u_{0}^{0}$ reduces to the classical Merton optimal consumption-investment problem. We shall use the dynamic programming approach in order to derive an explicit solution of the problem $u_{0}^{\alpha}$. We then need to introduce the dynamic version of this problem :

$$
u^{\alpha}(x, z):=\sup _{(C, \theta) \in \mathcal{A}_{\alpha}(x, z)} J(C, \theta),
$$

where the pair $(x, z)$, with $x \leq z$, stands for the initial condition of the state processes $(X, Z)$ defined, for $t \geq 0$, by

$$
Z_{t}^{x, z, C, \theta}:=z \vee\left\{X^{x, C, \theta}\right\}_{t}^{*} \text { and } X_{t}^{x, C, \theta}=x-\int_{0}^{t} C_{r} d r+\int_{0}^{t} \sigma \theta_{r}\left(d W_{r}+\lambda d r\right),
$$

and $\mathcal{A}_{\alpha}(x, z)$ is the collection of all $\mathbb{F}$-adapted processes $(C, \theta)$ satisfying $(2.3)$-(2.4) together with the drawdown constraint

$$
X_{t}^{x, C, \theta} \geq \alpha Z_{t}^{x, z, C, \theta}, \quad \text { for all } t \geq 0,
$$

almost surely. We also define $\mathcal{A}_{\alpha}^{0}(x, z)$ as the subset of consumption-investment strategies in $\mathcal{A}_{\alpha}(x, z)$ which satisfy the strict drawdown constraint

$$
X_{t}^{x, C, \theta}>\alpha Z_{t}^{x, z, C, \theta}, \quad \text { for all } t \geq 0
$$

almost surely. Clearly, avoiding the trivial case $x=z=0$, this restricts the pair of initial condition $(x, z)$ to the closure $\overline{\mathbf{D}}_{\alpha}$ in $(0, \infty) \times(0, \infty)$ of the domain

$$
\mathbf{D}_{\alpha}:=\{(x, z): 0<\alpha z<x \leq z\} .
$$

For completeness, we observe that the value function $u^{\alpha}$ can be easily shown to be concave with respect to the $x$ variable, see [19]. This result is not needed for the derivation of the solution.

\section{The verification result}

The optimal consumption-investment problem (2.17) is in the class of stochastic control problems studied in Barles, Daher and Romano [1]. The dynamic programming equation is related to the second order operator

$$
\mathcal{L} u:=-\beta u+\sup _{C \geq 0, \theta \in \mathbb{R}}\left[\mathcal{L}^{C, \theta} u+U(C)\right] \text { where } \mathcal{L}^{C, \theta} u:=(\theta \sigma \lambda-C) u_{x}+\frac{\theta^{2} \sigma^{2}}{2} u_{x x} .
$$

Our approach for the derivation of the explicit solution, reported in the next section, is based on the following verification result. 
Theorem 3.1 Let $w$ be a $C^{0}\left(\overline{\mathbf{D}}_{\alpha}\right) \cap C^{2,1}\left(\mathbf{D}_{\alpha}\right)$ function.

(i) If $w$ satisfies $-\mathcal{L} w \geq 0$ on $\mathbf{D}_{\alpha}$, and $-w_{z}(z, z) \geq 0$ for $z>0$, then $w \geq u^{\alpha}$.

(ii) Assume in addition that

1. $\mathcal{L} w=0$ on $\mathbf{D}_{\alpha}$, and $\min \left\{\beta w-V\left(w_{x}\right),-w_{z}\right\}(z, z)=0$ for $z>0$;

2. $\mathcal{L} w=-\beta w+U(\hat{C})+\mathcal{L}^{\hat{C}, \hat{\theta}} w$ on $\mathbf{D}_{\alpha}$, for some continuous functions $\hat{C}$ and $\hat{\theta}$, satisfying

$$
\hat{C}(\alpha z, z)=\hat{\theta}(\alpha z, z)=0, \quad z \geq 0
$$

and such that the stochastic differential equation

$$
d \hat{X}_{t}=-\hat{C}\left(\hat{X}_{t}, \hat{Z}_{t}\right) d t+\sigma \hat{\theta}\left(\hat{X}_{t}, \hat{Z}_{t}\right)\left(d W_{t}+\lambda d t\right) \quad t \geq 0, \quad \hat{Z}_{t}=z \wedge \hat{X}_{t}^{*},
$$

has a unique strong solution $(\hat{X}, \hat{Z})$ for any initial condition $\left(\hat{X}_{0}, \hat{Z}_{0}\right)=(x, z) \in \mathbf{D}_{\alpha}$ satisfying the strict drawdown constraint (2.20).

3. For every sequence of bounded stopping times $\left(\tau_{n}\right)_{n \geq 1}$ with $\tau_{n} \rightarrow \infty$ a.s., we have

$$
\liminf _{n \rightarrow \infty} \mathbb{E}\left[e^{-\beta \tau_{n}} w\left(\hat{X}_{\tau_{n}}, \hat{Z}_{\tau_{n}}\right)\right]=0 .
$$

Then, for $(x, z) \in \overline{\mathbf{D}}_{\alpha}$, we have $w(x, z)=u^{\alpha}(x, z)$ and $\left(C_{t}^{*}, \theta_{t}^{*}\right):=(\hat{C}, \hat{\theta})\left(\hat{X}_{t}, \hat{Z}_{t}\right)$ is an optimal consumption-investment strategy in $\mathcal{A}_{\alpha}^{0}(x, z)$ of the problem $u^{\alpha}(x, z)$.

For the proof of this result, we isolate the following lemma which is interesting on its own.

Lemma 3.1 For $z \geq 0$, we have $u^{\alpha}(\alpha z, z)=U(0) / \beta$, and the corresponding optimal consumption-investment strategies are given by $\left(C^{*}, \theta^{*}\right)=0$.

Proof. We first observe that for any $(C, \theta) \in \mathcal{A}_{\alpha}(\alpha z, z)$, the corresponding wealth process $X$ is bounded from below (as $X \geq \alpha Z \geq \alpha z$ ). Let $\mathbb{P}^{0}$ denote the equivalent martingale measure which turns the process $\left\{W_{t}^{0}:=W_{t}+\lambda t, t \geq 0\right\}$ into a $\mathbb{P}^{0}$-Brownian motion by the Girsanov theorem. Since the consumption process is nonnegative, this implies that the process $\left\{\int_{0}^{t} \sigma \theta_{r} d W_{r}^{0}, t \geq 0\right\}$ is a $\mathbb{P}^{0}$-supermartingale as a local martingale which is bounded from below.

By the dynamics of the wealth process, we have:

$$
\int_{0}^{t} C_{r} d r=\alpha z-X_{t}+\int_{0}^{t} \sigma \theta_{r} d W_{r}^{0}
$$

It then follows from the $\mathbb{P}^{0}$-supermartingale property of the process $\left\{\int_{0}^{t} \sigma \theta_{r} d W_{r}^{0}, t \geq 0\right\}$ together with the non-decrease of $X^{*}$ that

$$
\mathbb{E}^{\mathbb{P}^{0}}\left[\int_{0}^{t} C_{r} d r\right] \leq \mathbb{E}^{\mathbb{P}^{0}}\left[\alpha z-X_{t}\right]=\mathbb{E}^{\mathbb{P}^{0}}\left[\alpha X_{0}^{*}-X_{t}\right] \leq \mathbb{E}^{\mathbb{P}^{0}}\left[\alpha X_{t}^{*}-X_{t}\right] \leq 0,
$$

where the last inequality follows from the drawdown constraint. This shows that the only sustainable consumption from the initial condition $(\alpha z, z)$ is zero. We then deduce from (3.3) that

$$
\int_{0}^{t} \sigma \theta_{r} d W_{r}^{0}=X_{t}-\alpha z \geq 0
$$


which immediately implies the required result.

Proof of Theorem 3.1 We first observe that $-\mathcal{L} w \geq 0$ implies

$$
\beta w \geq V\left(w_{x}\right) \geq U(0)
$$

since $V$ is a decreasing function and $V(\infty)=U(0)$. From Lemma 3.1, this shows that $w \geq u^{\alpha}$ on $\overline{\mathbf{D}}_{\alpha} \backslash \mathbf{D}_{\alpha}$. Then, in case (ii), combining $-\mathcal{L} w=0$ with (3.2), we deduce $w=U(0) / \beta$ on $\overline{\mathbf{D}}_{\alpha} \backslash \mathbf{D}_{\alpha}$, and the statement of the theorem is trivial on $\overline{\mathbf{D}}_{\alpha} \backslash \mathbf{D}_{\alpha}$. From now on, we fix a pair $(x, z) \in \mathbf{D}_{\alpha}$.

(i) Let $(C, \theta)$ be an arbitrary admissible consumption-investment strategy in $\mathcal{A}_{\alpha}(x, z)$, set $(X, Z):=\left(X^{x, C, \theta}, Z^{x, z, C, \theta}\right)$ the solution of $(2.18)$ with initial condition $\left(X_{0}, Z_{0}\right)=(x, z)$, and define the non-decreasing sequence of stopping times

$$
\tau_{n}:=n \wedge \inf \left\{t>0: X_{t}-\alpha Z_{t}<n^{-1}\right\} .
$$

Denoting by $\tau_{\infty}$ its limit in the a.s. sense, it follows from the same argument as in the proof of Lemma 3.1 that

$$
\mathbf{1}_{\left\{\tau_{\infty}<\infty\right\}} \int_{\tau_{\infty}}^{\infty} C_{t} d t=0
$$

Observe now that, by Itô's formula, we obtain

$e^{-\beta \tau_{n}} w\left(X_{\tau_{n}}, Z_{\tau_{n}}\right)=w(x, z)+M_{n}+\int_{0}^{\tau_{n}} e^{-\beta t}\left(w_{z}\left(X_{t}, Z_{t}\right) d Z_{t}+\left(\mathcal{L}^{C_{t}, \theta_{t}} w-\beta w\right)\left(X_{t}, Z_{t}\right) d t\right)$

where

$$
M_{n}:=\int_{0}^{\tau_{n}} e^{-\beta t} \theta_{t} \sigma w_{x}\left(X_{t}, Z_{t}\right) d W_{t}, \quad n \geq 0 .
$$

Since $-w_{z}(z, z) \geq 0, Z$ is a non-decreasing process and $d Z_{t}=0$ whenever $X_{t}<Z_{t}$, it follows that the integral term with respect to $Z$ is non-negative. Using in addition the fact that $-\mathcal{L} w \geq 0$, we get

$$
w(x, z) \geq e^{-\beta \tau_{n}} w\left(X_{\tau_{n}}, Z_{\tau_{n}}\right)+\int_{0}^{\tau_{n}} e^{-\beta t} U\left(C_{t}\right) d t-M_{n} .
$$

Recall that $w_{x}$ is continuous on $\mathbf{D}_{\alpha}$. Then, it follows from the definition of $\tau_{n}$ that the stopped process $w_{x}(X, Z)$ is a.s. continuous on $\left[0, \tau_{n}\right]$. Since $\int_{0}^{n} \theta_{t}^{2} d t<\infty$ a.s., this implies that $M$ is a local martingale. By the lower bound (3.4) on $w$, it follows from (3.6) that $M$ is uniformly bounded from below. Then $M$ is a supermartingale. Taking expected values in (3.6), and using again the lower bound (3.4) on $w$, this implies that

$$
w(x, z) \geq \mathbb{E}\left[\int_{0}^{\tau_{n}} e^{-\beta t} U\left(C_{t}\right) d t+\frac{U(0)}{\beta} e^{-\beta \tau_{n}}\right] .
$$

By the monotone convergence theorem, this implies that

$$
w(x, z) \geq \mathbb{E}\left[\int_{0}^{\tau_{\infty}} e^{-\beta t} U\left(C_{t}\right) d t+\frac{U(0)}{\beta} e^{-\beta \tau_{\infty}} \mathbf{1}_{\left\{\tau_{\infty}<\infty\right\}}\right]=\mathbb{E}\left[\int_{0}^{\infty} e^{-\beta t} U\left(C_{t}\right) d t\right]
$$


by (3.5). Since $(C, \theta)$ is arbitrarily chosen in $\mathcal{A}_{\alpha}(x, z)$, this proves that $w(x, z) \geq u^{\alpha}(x, z)$.

(ii) We denote $\left(C_{t}^{*}, \theta_{t}^{*}\right):=(\hat{C}, \hat{\theta})\left(\hat{X}_{t}, \hat{Z}_{t}\right)$ and $\left(c_{t}^{*}, \pi_{t}^{*}\right):=\left(\hat{X}_{t}-\alpha \hat{Z}_{t}\right)^{-1}\left(C_{t}^{*}, \theta_{t}^{*}\right)$, for any $t \geq 0$. Since $(\hat{X}, \hat{Z})$ is assumed to satisfy the strict drawdown constraint, the sequence of bounded stopping times

$$
\hat{\tau}_{n}:=n \wedge \inf \left\{t>0: \hat{X}_{t}-\alpha \hat{Z}_{t}<n^{-1} \text { or } \hat{Z}_{t}>n\right\} \underset{n \rightarrow \infty}{\longrightarrow} \infty \text { a.s. }
$$

By Condition (ii-1) of the theorem together with the fact that $d Z_{t}=0$ whenever $X_{t}<Z_{t}$, it follows from Itô's lemma that

$$
w(x, z)=e^{-\beta \hat{\tau}_{n}} w\left(\hat{X}_{\hat{\tau}_{n}}, \hat{Z}_{\hat{\tau}_{n}}\right)+\int_{0}^{\hat{\tau}_{n}} e^{-\beta t} U\left(C_{t}^{*}\right) d t-\hat{M}_{n}
$$

where

$$
\hat{M}_{n}:=\int_{0}^{\hat{\tau}_{n}} e^{-\beta t} \sigma\left[\hat{\theta} w_{x}\right]\left(\hat{X}_{t}, \hat{Z}_{t}\right) d W_{t}, \quad n \geq 0
$$

Since $w_{x}$ and $\hat{\theta}$ are continuous on $\mathbf{D}_{\alpha}$, and the stopped process $(\hat{X}, \hat{Z})$ takes values in a compact subset of $\mathbf{D}_{\alpha}$, it follows that the process $\left(\hat{\theta} w_{x}\right)(\hat{X}, \hat{Z})$ is uniformly bounded on $\left[0, \hat{\tau}_{n}\right]$. Then $\hat{M}$ is a martingale, and

$$
w(x, z)=\mathbb{E}\left[e^{-\beta \hat{\tau}_{n}} w\left(\hat{X}_{\hat{\tau}_{n}}, \hat{Z}_{\hat{\tau}_{n}}\right)\right]+\mathbb{E}\left[\int_{0}^{\hat{\tau}_{n}} e^{-\beta t} U\left(C_{t}^{*}\right) d t\right]
$$

Since $\left(\hat{\tau}_{n}\right)_{n \geq 1}$ is a sequence of bounded stopping times converging to $\infty$ a.s., we may use Condition (ii-3) of the theorem which, together with the monotone convergence theorem, provides

$$
w(x, z)=\mathbb{E}\left[\int_{0}^{\infty} e^{-\beta t} U\left(C_{t}^{*}\right) d t\right]
$$

In view of (i), this implies that $w=u^{\alpha}$.

\section{Guessing a candidate solution of the dynamic program- ming equation}

In this section, we derive an explicit candidate solution of the optimal consumptioninvestment problem which satisfies the dynamic programming equation

$$
\begin{aligned}
& \mathcal{L} u(x, z)=0, \quad \text { for } \quad(x, z) \in \mathbf{D}_{\alpha} ; \\
& \min \left\{\beta u-V\left(u_{x}\right),-u_{z}\right\}(z, z)=0, \text { for } z>0 \text {. }
\end{aligned}
$$

which corresponds to Conditions (i) and (ii-1) of Theorem 3.1. Our main result in the subsequent Section 5 states that this candidate is indeed the value function of our problem of interest, and derives the corresponding optimal consumption and investment policy. This will be achieved by verifying the remaining conditions (ii-2)-(ii-3) of Theorem 3.1. 
We also observe that Condition (i) of Theorem 3.1 implies that $u_{x x} \leq 0$, i.e. the value function is expected to be concave. Then, defining the Fenchel-Legendre transform

$$
V(y):=\sup _{x \geq 0}(U(x)-x y)
$$

we re-write the nonlinear operator $\mathcal{L}$ defined in (3.1) into:

$$
\mathcal{L} u=\beta u-V\left(u_{x}\right)+\frac{\lambda^{2}}{2} \frac{u_{x}^{2}}{u_{x x}}
$$

with maximizers

$$
\hat{C}=-V^{\prime}\left(u_{x}\right)=\left(U^{\prime}\right)^{-1}\left(u_{x}\right) \text { and } \hat{\theta}:=-\frac{\lambda}{\sigma} \frac{u_{x}}{u_{x x}} .
$$

\subsection{The Fenchel-Legendre dual functions}

The key-ingredient in order to derive the explicit solution in this paper is to introduce the Fenchel-Legendre transforms of the value function $u^{\alpha}$ with fixed $z$ :

$$
v^{\alpha}(y, z):=\sup _{\alpha z \leq x \leq z}\left(u^{\alpha}(x, z)-x y\right) .
$$

Since the value function $u^{\alpha}$ is concave in its first variable, it can indeed be recovered from $v^{\alpha}$ by the duality relation

$$
u^{\alpha}(x, z)=\inf _{y \in \mathbb{R}}\left(v^{\alpha}(y, z)+x y\right)
$$

In the absence of drawdown constraint, the functions $u^{0}$ is independent of the $z$ variable and the dual function

$$
v^{0}(y):=\sup _{x \geq 0}\left(u^{0}(x)-x y\right)
$$

can be obtained explicitly in terms of the density of the risk-neutral measure. This can be seen by the following formal PDE argument: assuming that $u^{0}$ is smooth and satisfies the Inada conditions $\left(u^{0}\right)^{\prime}(0+)=+\infty,\left(u^{0}\right)^{\prime}(\infty)=0$, it follows that

$$
v^{0}(y)=u^{0}\left(\left[\left(u^{0}\right)^{\prime}\right]^{-1}(y)\right)-y\left[\left(u^{0}\right)^{\prime}\right]^{-1}(y) \text { for } y \geq 0
$$

and $v^{0}(y)=\infty$ for $y<0$. Substituting in the dynamic programming equation (4.1), it follows that $v^{0}$ solves on $(0, \infty)$ the linear parabolic partial differential equation

$$
\mathcal{L}^{*} v(y):=v(y)-y v_{y}(y)-\frac{1}{\gamma} y^{2} v_{y y}(y)=\frac{1}{\beta} V(y),
$$

where we have introduced the parameter

$$
\gamma:=\frac{2 \beta}{\lambda^{2}}
$$


which will play an important role throughout the paper. Under a convenient transversality condition, this provides

$$
v^{0}(y)=\mathbb{E}\left[\int_{0}^{\infty} e^{-\beta t} V\left(e^{\beta t} Y_{t}\right) d t\right] \text { where } Y_{t}:=y \exp \left(-\lambda W_{t}-\frac{1}{2} \lambda^{2} t\right) .
$$

This result is well-known in the financial mathematics literature, and can be proved rigourously by probabilistic arguments, see e.g. [12].

In this complete market setting, it is remarkable that the Fenchel transform $v^{0}$ solves a linear PDE. This is the key-observation in order to guess a candidate solution for the optimal consumption-investment problem under drawdown constraint.

\subsection{Guessing a candidate solution for the dual problem}

We now derive the PDE satisfied by the Fenchel-Legendre transform $v$ of a smooth solution $u$ of the dynamic programming equation (4.1)-(4.2).

Step 1: The PDE satisfied by the dual function $v$. We first introduce the functions

$$
\varphi(z):=u_{x}(z, z) \text { and } \psi(z):=u_{x}(\alpha z, z), \quad z>0 \text {. }
$$

For any $z>0$, since we are seeking for a concave function $u(., z)$, we expect that $\varphi(z) \leq$ $\psi(z)$. Denoting by $h(., z)$ the inverse of the function $u_{x}(., z)$, it follows from the definition of the dual function $v$ that

$$
\begin{aligned}
v(y, z)=u(h(y, z), z)-h(y, z) y & \text { if } \quad u_{x}(h(y, z), z)=y \in[\varphi(z), \psi(z)] \\
v(y, z)=u(z, z)-y z & \text { if } y \leq \varphi(z) \\
v(y, z)=u(\alpha z, z)-\alpha y z & \text { if } y \geq \psi(z) .
\end{aligned}
$$

Notice that $h(y, z)=-v_{y}(y, z)$ by the classical gradient correspondence from the Fenchel duality. In the situation of (4.11) where $y \in[\varphi(z), \psi(z)]$, we obtain by a direct change of variable in (4.1) that

$$
\mathcal{L}^{*} v(y, z)=V(y) \text { for } \varphi(z)<y<\psi(z)
$$

where $\mathcal{L}^{*}$ is the linear operator defined in (4.9). From the definition of the functions $\varphi$ and $\psi$, it follows from the Fenchel duality that

$$
\begin{aligned}
v_{y}(\varphi(z), z)=-z & \text { for } \quad z>0, \\
v_{y}(\psi(z), z)=-\alpha z & \text { for } \quad z>0 .
\end{aligned}
$$

We also observe that

- for $\varphi(z)<y<\psi(z)$, we have $\beta u-V\left(u_{x}\right)=\left(\beta\left(v-y v_{y}\right)-V\right) \circ u_{x}=\left(\lambda^{2} y^{2} v_{y y} / 2\right) \circ u_{x}$,

- and for $y<\varphi(z)$, we have from (4.12) that $v_{z}(y, z)=u_{x}(z, z)+u_{z}(z, z)-y$, and therefore $v_{z}(\varphi(z), z)=u_{z}(z, z)$ by the continuity of $v$ implied by its convexity and the definition of the function $\varphi$. 
Hence, the boundary condition (4.2) is converted into

$$
\min \left\{y v_{y y},-v_{z}\right\}(\varphi(z), z)=0 \text { for } z>0 .
$$

In the next step, we exhibit functions $v(y, z), \varphi(z), \psi(z)$ which satisfy the ODE (4.14) subject to the boundary conditions (4.15)-(4.16)-(4.17). We shall seek for such functions with

$$
\psi(z)=\infty \text { for every } z \geq 0,
$$

which is consistent with the economic intuition of the problem: when the drawdown constraint binds, the investor can neither consume nor invest for the next entire future, see Lemma 3.1, and therefore the marginal utility in this situation is expected to be infinite as $U^{\prime}(0)=\infty$. Notice that this is by no means an assumption we are making, as we only have to exhibit a candidate solution for which the conditions of our verification result, Theorem 3.1 hold.

Step 2: General solution of the ODE (4.14) subject to (4.15)-(4.16). The homogeneous equation, defined by ignoring the right hand-side of (4.14), has a linear space of solutions spanned by $v_{1}(y)=y$ and $v_{2}(y)=y^{-\gamma}$. A general solution for the linear ODE is derived by applying the technique of the variation of the constant. Direct calculations lead to

$$
v(y, z)=a(z) y-b(z) y^{-\gamma}+\frac{2}{\lambda^{2}(1+\gamma)} y \int_{\varphi(z)}^{\infty} V(\xi) \xi^{\gamma-1}(y \vee \xi)^{-(1+\gamma)} d \xi,
$$

where the latter integral is finite because $V(\infty)=U(0)<\infty$, and $a, b$ are functions of $z$ to be determined by using the boundary conditions. Direct calculation leads to

$$
v_{y}(y, z)=a(z)+\gamma b(z) y^{-(1+\gamma)}+\frac{2}{\lambda^{2}(1+\gamma)}\left(-\gamma y^{-(1+\gamma)} \int_{\varphi(z)}^{y} V(\xi) \xi^{\gamma-1} d \xi+\int_{y}^{\infty} V(\xi) \xi^{-2} d \xi\right) .
$$

In view of (4.18), it follows from (4.16) that $a(z)=-\alpha z$. We also easily express the function $b(z)$ in terms of $\varphi(z)$ by using the boundary condition (4.15), and we update our candidate solution:

$$
\begin{aligned}
v(y, z)= & -\alpha y z+\frac{y}{\gamma}\left(\frac{\varphi(z)}{y}\right)^{1+\gamma}\left((1-\alpha) z+\frac{2}{\lambda^{2}(1+\gamma)} \int_{\varphi(z)}^{\infty} V(\xi) \xi^{-2} d \xi\right) \\
& +\frac{2}{\lambda^{2}(1+\gamma)}\left(y^{-\gamma} \int_{\varphi(z)}^{y} V(\xi) \xi^{\gamma-1} d \xi+y \int_{y}^{\infty} V(\xi) \xi^{-2} d \xi\right)
\end{aligned}
$$

Step 3: Determining the marginal utility at the maximum $\varphi(z)$. We now make use of the boundary condition (4.17) in order to determine the function $\varphi$. Direct computation provides:

$$
\varphi(z) v_{y y}(\varphi(z), z)=(1+\gamma)(1-\alpha) z-\frac{2}{\lambda^{2}} \int_{\varphi(z)}^{\infty}-V^{\prime}(\xi) \xi^{-1} d \xi
$$

and

$$
v_{z}(\varphi(z), z)=\left(\frac{1-\alpha}{\gamma}-\alpha\right) \varphi(z)+\frac{\varphi^{\prime}(z)}{\gamma}\left[(1+\gamma)(1-\alpha) z-\frac{2}{\lambda^{2}} \int_{\varphi(z)}^{\infty}-V^{\prime}(\xi) \xi^{-1} d \xi\right] .
$$


We continue our calculation by postulating that

$$
\varphi(z)>0 \text { and } \varphi^{\prime}(z)<0 \text { for all } z>0
$$

a claim which will be verified by our candidate solution below. Then, the boundary condition (4.17) is equivalent to

$$
(1+\gamma)(1-\alpha) z-\frac{2}{\lambda^{2}} \int_{\varphi(z)}^{\infty}-V^{\prime}(\xi) \xi^{-1} d \xi=-(1-\alpha(1+\gamma))^{+} \frac{\varphi(z)}{\varphi^{\prime}(z)} .
$$

Introducing the inverse $g:=\varphi^{-1}$ which is well-defined under (4.21), we re-write the latter equation into:

$$
g(\zeta)-\phi(\zeta)=-\frac{(1-\alpha[1+\gamma])^{+}}{(1-\alpha)(1+\gamma)} \zeta g^{\prime}(\zeta)
$$

where we have denoted

$$
\phi(\zeta):=\frac{2}{\lambda^{2}(1-\alpha)(1+\gamma)} \int_{\zeta}^{\infty}-\frac{V^{\prime}(\xi)}{\xi} d \xi \text { for } \zeta>0
$$

This leads to the two following cases.

Case $A \quad \alpha \geq(1+\gamma)^{-1}$, then the expression of $g$ is immediately given by the last equation:

$$
g(\zeta)=\phi(\zeta) \text { for } \quad \zeta>0
$$

Notice that this function is a decreasing map from $(0, \infty)$ in $(0, \infty)$, thus verifying $(4.21)$ for its inverse $\varphi$.

$\underline{\text { Case } B} \quad \alpha<(1+\gamma)^{-1}$, then it follows from (4.22) that $g$ satisfies the first order linear $\mathrm{ODE}$

$$
g(\zeta)+\frac{1-\alpha(1+\gamma)}{(1+\gamma)(1-\alpha)} \zeta g^{\prime}(\zeta)=\phi(\zeta)
$$

which provides a unique non-negative solution, as required by (4.21):

$$
\begin{aligned}
g(\zeta) & =\phi(\zeta)+\frac{2}{\lambda^{2}(1-\alpha)(1+\gamma)} \int_{0}^{\zeta} \frac{-V^{\prime}(\xi)}{\xi}\left(\frac{\xi}{\zeta}\right)^{\frac{(1-\alpha)(1+\gamma)}{1-\alpha(1+\gamma)}} d \xi \\
& =\frac{2}{\lambda^{2}(1-\alpha)(1+\gamma)} \int_{0}^{\infty} \frac{-V^{\prime}(\xi)}{\xi}\left(1 \wedge \frac{\xi}{\zeta}\right)^{\frac{(1-\alpha)(1+\gamma)}{1-\alpha(1+\gamma)}} d \xi
\end{aligned}
$$

where the second equality follows by interchanging the order of integration. The integrability of the latter expression will be ensured by a condition on the asymptotic elasticity of $U$, see Remark 5.1 below. Furthermore, it is now clear that $g$ is a decreasing map from $(0, \infty)$ to $(0, \infty)$, thus implying that $(4.21)$ holds true. 


\subsection{Candidate solution to the consumption-investment problem}

We now use the explicit form (4.20) of the dual function $v$ in order to derive an explicit candidate solution for the consumption-investment problem under drawdown constraint. By the duality relation between $u$ and $v$, we have

$$
u(x, z)=v \circ f(x, z)+x f(x, z)
$$

where $f(., z)$ is the inverse function of $h(., z)=-v_{y}(., z)$ given by

$$
h(y, z)=\alpha z+(1-\alpha)[z-\phi(\varphi(z))]\left(\frac{\varphi(z)}{y}\right)^{1+\gamma}+\frac{2}{\lambda^{2}(1+\gamma)} \int_{\varphi(z)}^{\infty} \frac{-V^{\prime}(\xi)}{\xi}\left(\frac{y \wedge \xi}{y}\right)^{1+\gamma} d \xi
$$

and we recall from the previous subsection that $\varphi=g^{-1}$ is implicitly defined by:

$$
\frac{\lambda^{2}}{2}(1-\alpha)(1+\gamma) z=\int_{\varphi(z)}^{\infty}-\frac{V^{\prime}(\xi)}{\xi} d \xi+1_{\{\alpha(1+\gamma)<1\}} \int_{0}^{\varphi(z)} \frac{-V^{\prime}(\xi)}{\xi}\left(\frac{\xi}{\varphi(z)}\right)^{\frac{(1-\alpha)(1+\gamma)}{1-\alpha(1+\gamma)}} d \xi
$$

The invertibility of the above function $h(., z)$ is clear from the expression (4.27) together with the fact that $z-\phi(\varphi(z)) \geq 0$ implied by (4.28). Thus its inverse function $f(., z)$ is a strictly decreasing function from $(\alpha z, z]$ to $[\varphi(z), \infty)$ defined implicitly by

$$
\begin{aligned}
\frac{\lambda^{2}}{2}(1+\gamma)(x-\alpha z)= & \int_{\varphi(z)}^{\infty} \frac{-V^{\prime}(\xi)}{\xi}\left(\frac{f(x, z) \wedge \xi}{f(x, z)}\right)^{1+\gamma} d \xi \\
& +\mathbf{1}_{\{\alpha(1+\gamma)<1\}}\left(\frac{\varphi(z)}{f(x, z)}\right)^{1+\gamma} \int_{0}^{\varphi(z)} \frac{-V^{\prime}(\xi)}{\xi}\left(\frac{\xi}{\varphi(z)}\right)^{\frac{(1-\alpha)(1+\gamma)}{1-\alpha(1+\gamma)}} d \xi
\end{aligned}
$$

Combining (4.26) with expressions (4.20), (4.28) and (4.29), some direct computation shows that the candidate value function $u$ is given by:

$$
u(x, z)=f(x, z)\left(\frac{1+\gamma}{\gamma}(x-\alpha z)+\frac{1}{\beta} \int_{f(x, z)}^{\infty} V(\xi) \xi^{-2} d \xi\right) \quad \text { for } \quad(x, z) \in \mathbf{D}_{\alpha} .
$$

The candidate optimal consumption-investment strategy is directly obtained by means of the maximizer (4.5) in the dynamic programming equation, as

$$
\hat{C}(x, z)=-V^{\prime}(f(x, z)), \quad(x, z) \in \mathbf{D}_{\alpha},
$$

and

$$
\hat{\theta}(x, z)=\frac{\lambda}{\sigma}(1+\gamma)(x-\alpha z)-\frac{2}{\lambda \sigma} \int_{f(x, z)}^{\infty}-V^{\prime}(\xi) \xi^{-1} d \xi, \quad(x, z) \in \mathbf{D}_{\alpha}
$$

Since $f(\alpha z, z)=\infty$, we finally observe that the optimal strategy vanishes on the drawdown boundary $\overline{\mathbf{D}}_{\alpha} \backslash \mathbf{D}_{\alpha}$ :

$$
\hat{\theta}(\alpha z, z)=\hat{C}(\alpha z, z)=0, \quad z \geq 0,
$$

in agreement with lemma (3.1). Further analysis of the behavior of $f$ will also reveal that

$$
u(\alpha z, z)=\frac{U(0)}{\beta}, \quad z \geq 0,
$$

see Proposition 6.1. 


\section{The main result}

This section reports our main result stating that the candidates (4.30)-(4.31)-(4.32) are indeed the value function of our consumption-investment problem under drawdown constraint (2.17) and the corresponding optimal consumption and investment policy. We first recall the notion of asymptotic elasticity introduced by Kramkov and Schachermayer [15]:

$$
\mathrm{AE}(U):=\limsup _{x \rightarrow \infty} \frac{x U^{\prime}(x)}{U(x)}
$$

Theorem 5.1 Let the utility function $U$ satisfy (2.15), and assume that

$$
\mathrm{AE}(\mathrm{U})<1 \wedge \frac{\gamma}{(1-\alpha)(1+\gamma)}
$$

Then $u^{\alpha}$ coincides with the candidate defined by (4.30)-(4.34). Moreover, $u^{\alpha}$ is a $C^{0}\left(\overline{\mathbf{D}}_{\alpha}\right) \cap$ $C^{2,1}\left(\mathbf{D}_{\alpha}\right)$ function, and for any initial data $\left(\hat{X}_{0}, \hat{Z}_{0}\right)=(x, z) \in \overline{\mathbf{D}}_{\alpha}$, the stochastic differential equation

$$
d \hat{X}_{t}=-\hat{C}\left(\hat{X}_{t}, \hat{Z}_{t}\right) d t+\hat{\theta}\left(\hat{X}_{t}, \hat{Z}_{t}\right) \sigma\left(d W_{t}+\lambda d t\right), \hat{Z}_{t}:=z \vee \hat{X}_{t}^{*},
$$

has a unique strong solution with values $\left(\hat{X}_{t}, \hat{Z}_{t}\right) \in \mathbf{D}_{\alpha}$ a.s., and the pair process $\left(C^{*}, \theta^{*}\right):=$ $(\hat{C}, \hat{\theta})(\hat{X}, \hat{Z}) \in \mathcal{A}_{\alpha}^{0}(x, z)$ is a solution of the problem.

This theorem is proved by verifying that the candidate $u$ satisfies all the conditions of the verification Theorem 3.1. The rather technical proof of the result is reported to Section 6. We outline that the particular case of a power utility function, treated in Section 6.3.2, is of interest in itself since the form of the corresponding value function is much more tractable. In particular, as explained in the next remark, it provides a convenient control on the growth of the candidate value function $u$, used to verify the transversality condition (ii-3) of Theorem 3.1.

Remark 5.1 By Lemma 6.5 in [15], it follows from the condition on the asymptotic elasticity of $U$ that there exists a constant $K_{p}$ such that

$$
U(x) \leq K_{p}\left(1+\frac{x^{p}}{p}\right), \quad x \geq 0, \text { where } p:=\mathrm{AE}(U) .
$$

Furthermore, since $U$ and $V$ satisfy the relation

$$
U(x)=V\left(\left(-V^{\prime}\right)^{-1}(x)\right)+x\left(-V^{\prime}\right)^{-1}(x), \quad x \geq 0,
$$

where both terms on the right hand side are positive, it follows from (5.2) together with the fact that $U^{\prime}(\infty)=0$ that is

$$
\limsup _{y \rightarrow 0}-V^{\prime}(y) y^{\frac{1}{1-p}}<\infty .
$$

This condition ensures the existence of the integral terms in (4.28) and (4.29) and imply that our candidates are well defined. 


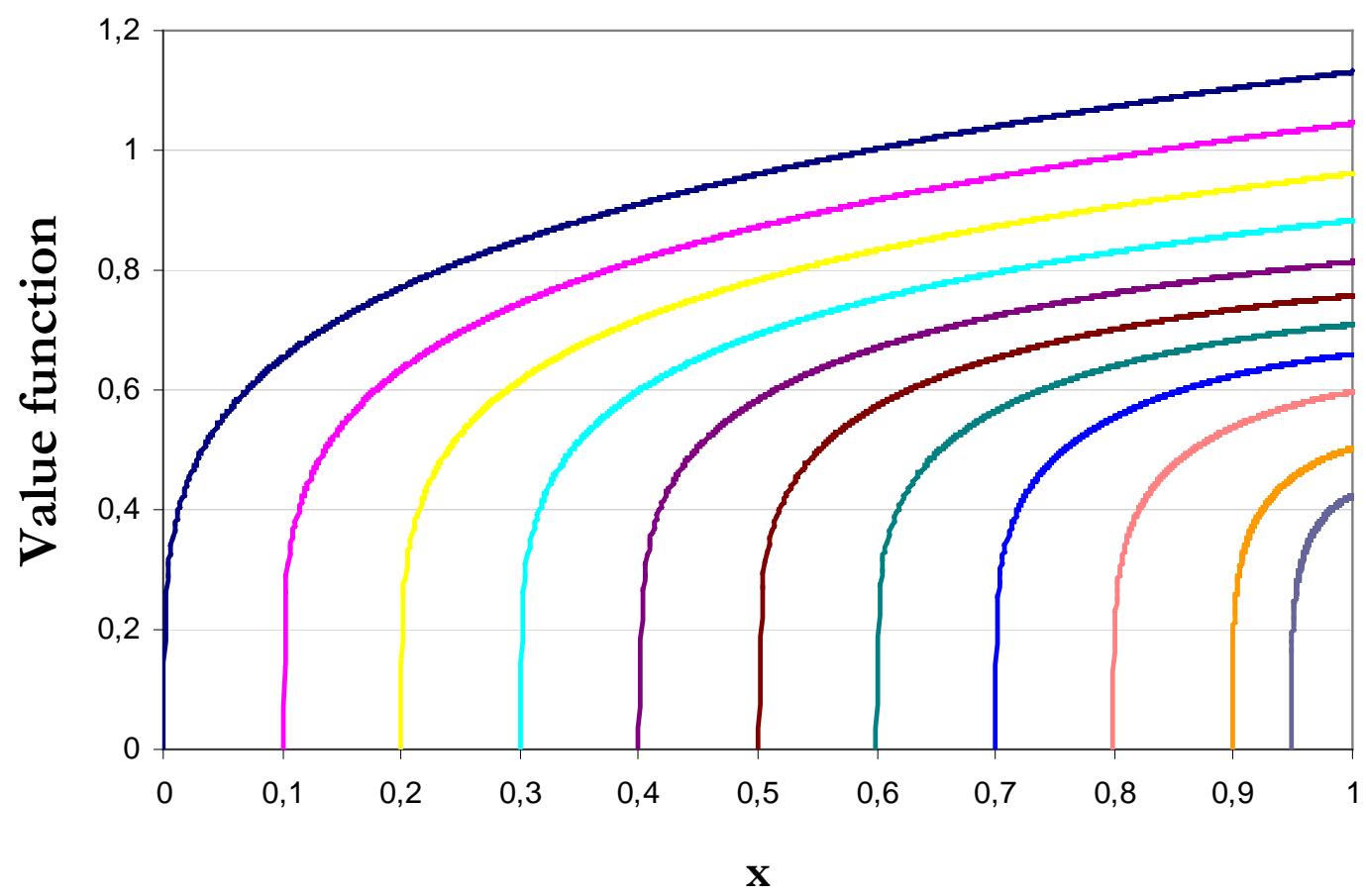

Figure 1: Value function $u^{\alpha}(x, 1)$ versus the wealth $x$ for different $\alpha$

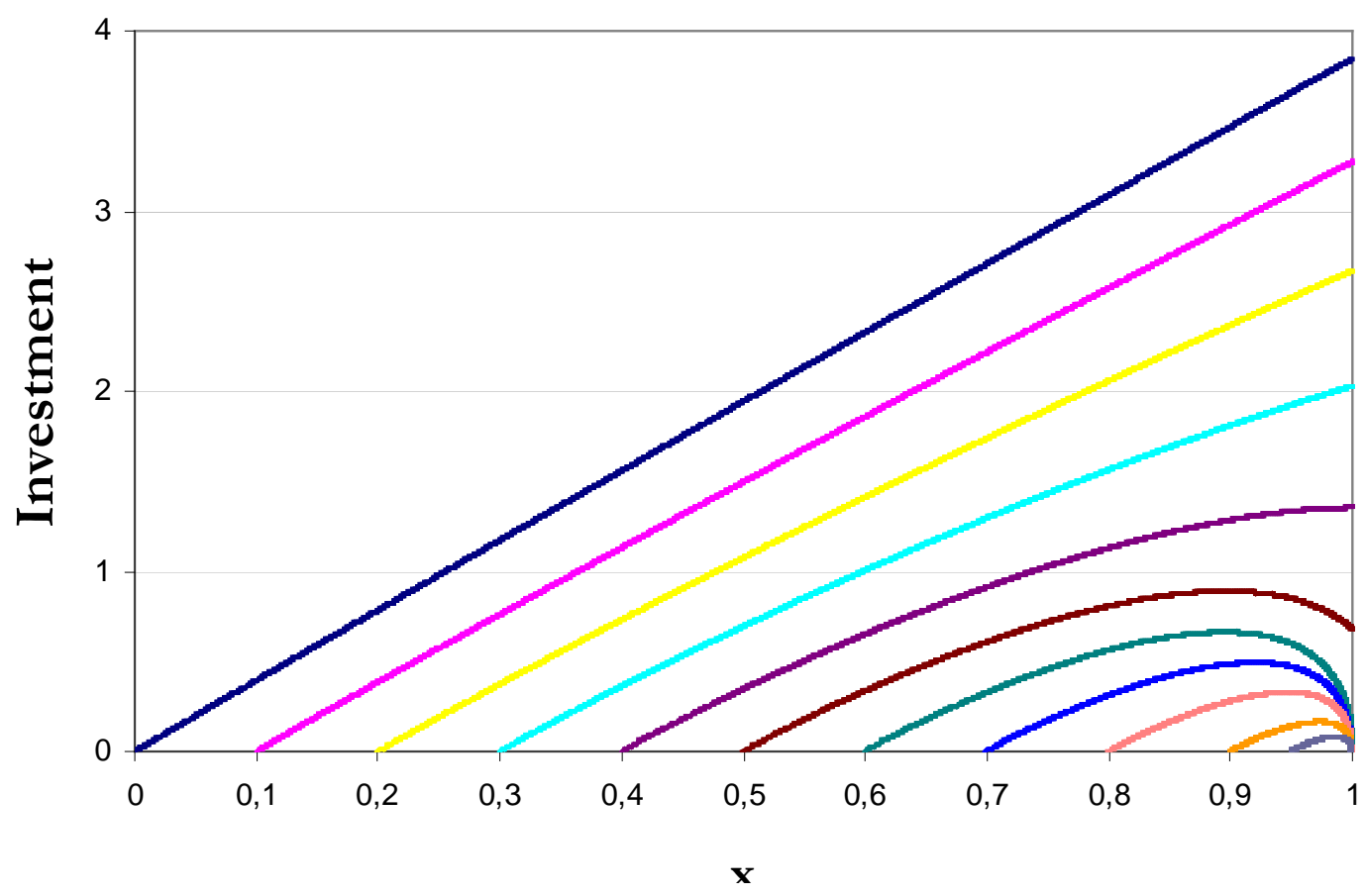

Figure 2: Investment $\theta$ versus the wealth $x$ for different $\alpha$ 
Remark 5.2 From the expression (4.32) of the optimal investment policy together with (4.28), we see that

$$
\hat{\theta}(z, z)=0, \text { for } z \geq 0 \text { if and only if } \alpha \geq(1+\gamma)^{-1},
$$

so that the dynamics of the optimal wealth process on $\left\{\hat{X}_{t}=\hat{Z}_{t}\right\}$ is $d \hat{X}_{t}=-C_{t}^{*} d t$. Since the consumption process is non-negative, this means that the optimal wealth process decreases immediatey when it hits the current maximum $\hat{Z}$. In other words, the process $\hat{Z}$. $=\hat{Z}_{0}$ is flat. This behavior was already observed in [19] and shows that for a large drawdown parameter $\alpha$, the investor anticipates that reaching its current maximum of wealth will increase the floor imposed by the drawdown constraint, and therefore chooses to consume instead of investing in the risky asset. This observation is consistent with the results of Roche [19]. Notice however that the optimal investment strategy derived by [9] does not exhibit this behavior, as their model considers an agent maximizing the long term growth rate of expected utility of its final wealth, with no utility from intermediate consumption.

We conclude this section by reporting some numerical examples in the case of the utility function

$$
U(x):=x^{p_{1}}+x^{p_{2}}, \quad x \geq 0,
$$

with $p_{1}=0.2$ and $p_{2}=0.3$. Our main objective is to comment on the effect of the drawdown parameter $\alpha \in[0,1)$. We therefore consider the following set of parameters $(\sigma, \lambda, \beta)=(1,3,3)$ which statisfies the asymptotic elasticity condition of the theorem for all $\alpha \in[0,1)$, i.e. for $\alpha=0$. In the following figures we plot the value function, the optimal investment strategy and the optimal consumption strategy for various values of $\alpha$ and for fixed $z=1$. Since these functions are defined on $[\alpha, 1]$, the value of the drawdown parameter $\alpha$ is identified on the graph as the starting point of the curve on the $x$-axis.

As expected, Figure 1 shows that the value function $u^{\alpha}$ is decreasing in $\alpha$. Figure 2 shows that investment in the risky asset decreases with $\alpha$. We also observe that near $x=\alpha z$, i.e. when the drawdown constraint nearly binds, the slope does not depend on $\alpha$. We next comment on the behavior of the optimal investment strategy on both sides of the threshold $\alpha=(1+\gamma)^{-1}$ :

- When $\alpha<(1+\gamma)^{-1}=0.6$, the curves $\hat{\theta}(., 1)$ are clearly Lipschitz in $x$ in agreement with the subsequent Lemma 6.2. For large wealth $x$, the agent is more reluctant to invest in the risky asset as $\alpha$ increases. When the wealth process approaches its maximum, the amount invested in the risky asset even decreases for large $\alpha$. The optimal consumption strategy, reported in Figure 3, is increasing in $\alpha$ for large values of wealth while it is decreasing otherwise.

- When $\alpha \geq(1+\gamma)^{-1}=0.6$, the curves $\hat{\theta}(., 1)$ are only locally Lipschitz in $x$ as the gradient near $x=1$ approaches $\infty$. This is again in agreement with the subsequent Lemma 6.2. We also observe that $\hat{\theta}(1,1)=0$ implying that the current maximum process of the optimal wealth is flat, see Remark 5.2. In other words since $Z_{0}=X_{0}$ is fixed, this case reduces to a problem of optimal consumption-investment with state constraint $X . \in\left[\alpha X_{0}, X_{0}\right]$ on the wealth process. As for the optimal consumption strategy, it is now clearly decreasing in $\alpha$. 


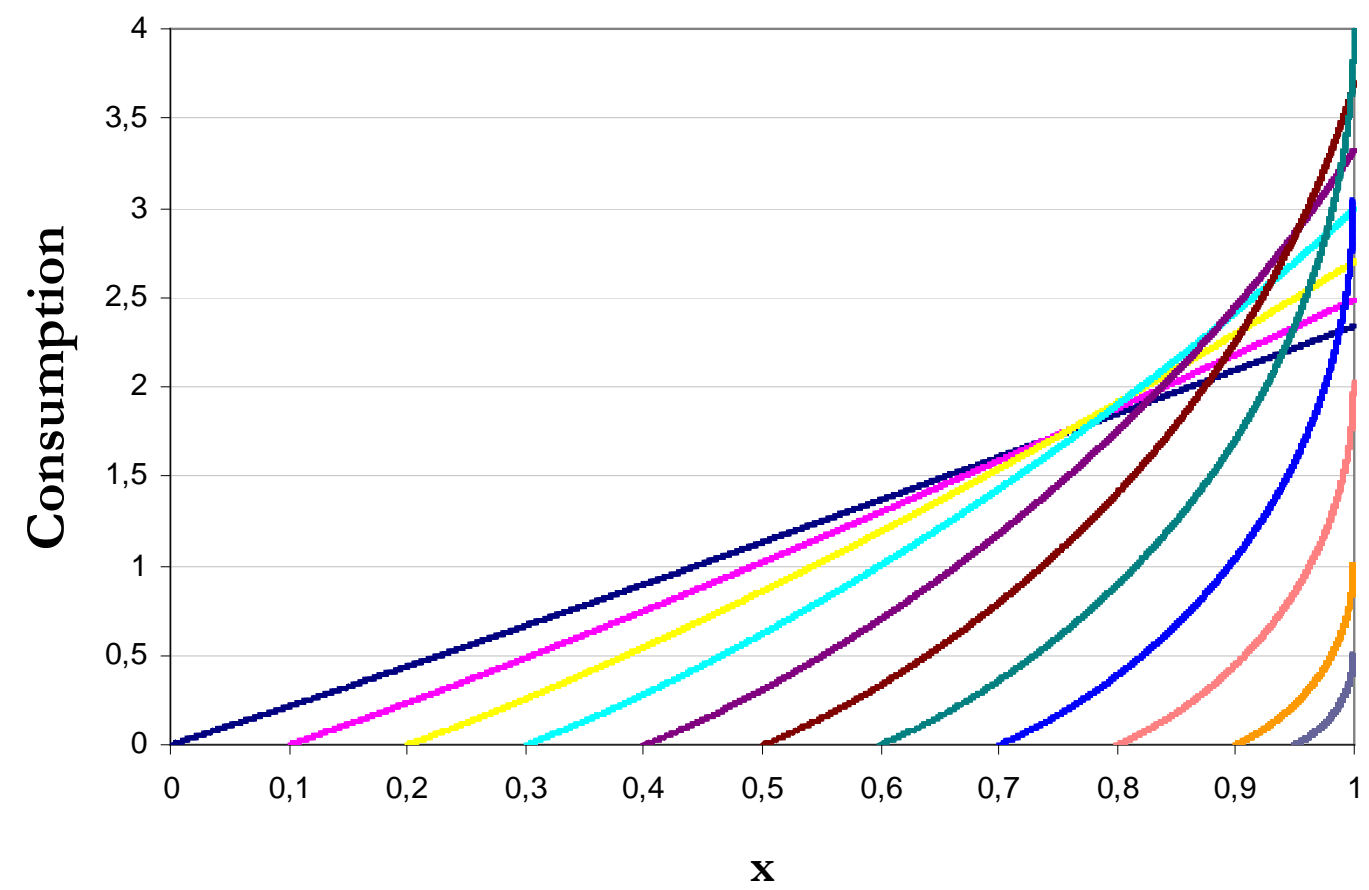

Figure 3: Consumption $C(x, 1)$ versus the wealth $x$ for different $\alpha$

\section{Proof of Theorem 5.1}

This section is devoted to the proof of Theorem 5.1. We shall check the candidate value function $u$ and the optimal consumption-investment strategy $(\hat{C}, \hat{\theta})$ defined by (4.30)-(4.31)(4.32) satisfy all the hypothesis of the verification theorem 3.1. The regularity property and Condition (ii-1) are given by Proposition 6.1, and conditions (ii-2) and (ii-3) follow respectively from Proposition 6.2 and Proposition 6.3.

Throughout this section, the utility function is assumed to satisfy the asymptotic elasticity condition of Theorem 5.1:

$$
\mathrm{AE}(\mathrm{U})<1 \wedge \frac{\gamma}{(1-\alpha)(1+\gamma)}
$$

In particular, this ensure that the candidates solution $u$ of (4.30)-(4.31)-(4.32) are well defined, see Remark 5.1.

\subsection{Regularity of the candidate value function}

The following proposition collects the regularity properties of the candidate function $u$ given by (4.30), needed for the application of the verification Theorem 3.1. By construction, this ensures that the candidate solution $u$ is a classical solution of the dynamic programming equation (4.1)-(4.2). 
Proposition 6.1 The candidate value function $u \in C^{0}\left(\overline{\mathbf{D}}_{\alpha}\right) \cap C^{2,1}\left(\mathbf{D}_{\alpha}\right)$ and satisfies

$$
\mathcal{L} u=0 \text { on } \mathbf{D}_{\alpha}, \quad \text { and } \min \left\{\beta u-V\left(u_{x}\right),-u_{z}\right\}(z, z)=0, \quad \text { for } z>0 .
$$

The proof of this proposition requires the use of the following regularity result on the function $f$ defined implicitly by (4.29).

Lemma 6.1 The function $f \in C^{1}\left(\mathbf{D}_{\alpha}\right)$ and, for any $(x, z) \in \mathbf{D}_{\alpha}$, we have :

$$
\begin{aligned}
& \frac{f_{x}(x, z)}{f(x, z)}=-\left((\gamma+1)(x-\alpha z)+\frac{\gamma}{\beta} \int_{f(x, z)}^{\infty} \frac{V^{\prime}(s)}{s} d s\right)^{-1}, \\
& \frac{f_{z}(x, z)}{f(x, z)}=\alpha\left(1+\left[\gamma \wedge \frac{1-\alpha}{\alpha}\right]\left(\frac{\varphi(z)}{f(x, z)}\right)^{\gamma+1}\right) \frac{f_{x}(x, z)}{f(x, z)} .
\end{aligned}
$$

Proof. By construction, the function $f$ defined as the inverse of $h$ satisfies

$$
f(h(y, z), z)=y, \text { for } y \geq \varphi(z), \text { and } h(f(x, z), z)=x, \text { for }(x, z) \in \mathbf{D}_{\alpha} \text {. }
$$

By definition, the function $h(., z)$ and its inverse $f(., z)$ are $C^{1}$ and decreasing, for any $z>0$. Direct computation then leads to (6.3). We also observe that $h \in C^{1,1}(\{(y, z), y \geq \varphi(z)\})$ and that we have

$$
0 \leq h_{z}(y, z)=\alpha+[\alpha \gamma \wedge(1-\alpha)]\left(\frac{\varphi(z)}{y}\right)^{\gamma+1} \leq \alpha(1+\gamma), \quad y \geq \varphi(z)
$$

Therefore, $h$ and $f$ are increasing in $z$. Collecting the monotonicity properties of $f$, we have:

$$
f \text { is decreasing in } x \text {, increasing in } z \text {, and } \varphi: z \mapsto f(z, z) \text { is decreasing, }
$$

recall that $\varphi$ satisfies (4.21). In order to prove that $f \in C^{1}\left(D_{\alpha}\right)$, we shall prove that $f$ is differentiable in each variable with continuous partial derivatives.

1. In this step, we show that $f \in C^{0}\left(\mathbf{D}_{\alpha}\right)$, which implies that $f_{x} \in C^{0}\left(\mathbf{D}_{\alpha}\right)$ by (6.3). For $(x, z) \in \mathbf{D}_{\alpha}$, we study separately two alternative cases:

- If $x<z$, for $l^{\prime}$ small enough, $\left(x, z+l^{\prime}\right) \in \mathbf{D}_{\alpha}$ and we deduce from (6.6) that

$$
h\left(f\left(x, z+l^{\prime}\right), z\right)-x=h\left(f\left(x, z+l^{\prime}\right), z\right)-h\left(f\left(x, z+l^{\prime}\right), z+l^{\prime}\right) \leq \alpha(1+\gamma) l^{\prime} \underset{l^{\prime} \rightarrow 0}{\longrightarrow} 0 .
$$

Therefore, since $f\left(x, z+l^{\prime}\right) \geq \varphi(z)$ from the monotonicity of $f$, combining (6.5) and the continuity of $f(., z)$, we obtain

$$
f\left(x, z+l^{\prime}\right)-f(x, z)=f\left(h\left(f\left(x, z+l^{\prime}\right), z\right), z\right)-f(x, z) \underset{l^{\prime} \rightarrow 0}{\longrightarrow} 0 .
$$

Moreover, notice that $\left(x+l, z+l^{\prime}\right) \in \mathbf{D}_{\alpha}$ for sufficiently small $l$, and we have

$$
f\left(x+l, z+l^{\prime}\right)-f(x, z)=f_{x}\left(x_{l}, z+l^{\prime}\right) l+f\left(x, z+l^{\prime}\right)-f(x, z)
$$


for some $x_{l} \in[x, x+l]$. Now, since $f$ is monotonic in both variables, we deduce from (6.3) that $f$ and $f_{x}$ are bounded on any compact subset of $\mathbf{D}_{\alpha}$ containing $(x, z)$. Therefore, combining (6.7) and (6.8), we deduce that $f$ is continuous at point $(x, z)$.

- If $x=z$, we have, for any $l$ and $l^{\prime}$ satisfying $\left(z+l, z+l^{\prime}\right) \in \mathbf{D}_{\alpha}$,

$$
f\left(z+l, z+l^{\prime}\right)=f_{x}\left(z_{l}, z+l^{\prime}\right)\left(l^{\prime}-l\right)+\varphi\left(z+l^{\prime}\right), \text { for some } z_{l} \in\left[z+l, z+l^{\prime}\right] .
$$

Therefore similar arguments as above combined with the continuity of $\varphi$ lead to the continuity of $f$ on $\mathbf{D}_{\alpha}$.

2. We now prove that $f$ is differentiable with respect to $z$ with continuous partial derivatives. Take $(x, z) \in \mathbf{D}_{\alpha}$ and $l^{\prime}$ such that $\left(x, z+l^{\prime}\right) \in \mathbf{D}_{\alpha}$. Combining $f(x, z) \geq \varphi\left(z+l^{\prime}\right)$ with (6.5), we deduce

$$
\begin{aligned}
\frac{1}{l^{\prime}}\left\{f\left(x, z+l^{\prime}\right)-f(x, z)\right\} & =\frac{1}{l^{\prime}}\left\{f\left(x, z+l^{\prime}\right)-f\left(h\left(f(x, z), z+l^{\prime}, z+l^{\prime}\right)\right)\right\} \\
& =f_{x}\left(x_{l^{\prime}}, z+l^{\prime}\right) \frac{1}{l^{\prime}}\left\{h(f(x, z), z)-h\left(f(x, z), z+l^{\prime}\right)\right\},
\end{aligned}
$$

for some $x_{l^{\prime}} \in\left[x, x+l^{\prime}\right]$. Since $f_{x} \in C^{0}\left(\mathbf{D}_{\alpha}\right)$ and $h_{z}(f(x, z),$.$) is continuous, we obtain$

$$
\frac{1}{h^{\prime}}\left\{f\left(x, z+h^{\prime}\right)-f(x, z)\right\} \underset{h^{\prime} \rightarrow 0}{\longrightarrow}-f_{x}(x, z) h_{z}(f(x, z), z) .
$$

Finally, combining (6.3) and (6.6), simple computations lead to (6.4) and $f_{z}$ inherits the continuity of $f$ on $\mathbf{D}_{\alpha}$.

We are now ready for the

Proof of Proposition 6.1 We only need to prove the regularity properties of the candidate value function $u$, as (6.2) is satisfied by the construction of $u$ in Section 4.2.

By Lemma 6.1, $f \in C^{1}\left(\mathbf{D}_{\alpha}\right)$, and we deduce from (4.30) that $u \in C^{1}\left(\mathbf{D}_{\alpha}\right)$. Direct computation leads to $u_{x}=f$ on $\mathbf{D}_{\alpha}$ and therefore $u \in C^{2,1}\left(\mathbf{D}_{\alpha}\right)$. We now prove that $u \in C^{0}\left(\overline{\mathbf{D}}_{\alpha}\right)$.

Combining (4.30) with the definition of $f$, it follows from an integration by part argument,

$$
\begin{aligned}
u(x, z) & =\frac{\gamma}{\beta} \int_{\varphi(z)}^{f(x, z)} \frac{V(s)}{s}\left(\frac{s}{f(x, z)}\right)^{\gamma} d s+V \circ \varphi(z)\left(\frac{\varphi(z)}{f(x, z)}\right)^{\gamma} \\
& +\mathbf{1}_{\left\{\alpha<(1+\gamma)^{-1}\right\}} \frac{2}{\lambda^{2}(1-\alpha)(1+\gamma)} \int_{0}^{\zeta} \frac{-V^{\prime}(\xi)}{\xi}\left(\frac{\xi}{\zeta}\right)^{\frac{(1+\alpha)(1+\gamma)}{1-\alpha(1+\gamma)}} d \xi, \quad(x, z) \in \mathbf{D}_{\alpha} .
\end{aligned}
$$

Since the function $V^{\prime}$ is negative, $V$ is bounded from below by $V(\infty)=U(0)$, and we deduce from (6.9) that $u \geq U(0) / \beta$.

Fix now $z_{0}>0, \epsilon>0$ and let $\mathcal{C}_{0}$ be a compact subset of $\mathbb{R}^{+}$containing $z_{0}$. Observe that there exists a constant $M$ such that $|V(y)-U(0)| \leq \beta \epsilon / 2$ for $y \geq M$. Since $\varphi$ and $V$ are continuous functions and therefore bounded on compact sets, we deduce from (6.9) the existence of a constant $K>0$ satisfying

$$
u(x, z) \leq\left(\frac{K}{f(x, z)}\right)^{\gamma}+\frac{U(0)}{\beta}+\frac{\epsilon}{2}, \quad(x, z) \in \mathbf{D}_{\alpha}, z \in \mathcal{C}_{0} .
$$


Observe now that, since $V^{\prime}$ is a negative function, we derive from (6.3),

$$
-\frac{f_{x}(x, z)}{f(x, z)} \geq \frac{1}{(\gamma+1)(x-\alpha z)}, \quad(x, z) \in \mathbf{D}_{\alpha} .
$$

Integrating this inequality on the interval $[x, z]$, we obtain, up to the composition with the exponential function,

$$
f(x, z) \geq \varphi(z)[(1-\alpha) z]^{1 /(1+\gamma)}(x-\alpha z)^{-1 /(1+\gamma)}, \quad(x, z) \in \mathbf{D}_{\alpha} .
$$

Therefore, there exists $\eta>0$ such that, for any $(x, z) \in \mathbf{D}_{\alpha}$ with $z \in \mathcal{C}_{0}$ and $|x-\alpha z|<\eta$, we have $f(x, z)>K(\epsilon / 2)^{-1 / \gamma}$, which plugged in (6.10) leads to

$$
\frac{U(0)}{\beta} \leq u(x, z) \leq \frac{U(0)}{\beta}+\epsilon .
$$

Since this is true for any $\varepsilon>0, u \in C^{0}\left(\overline{\mathbf{D}}_{\alpha}\right)$.

\subsection{The wealth process with optimal feedback policy}

Given an initial condition $(x, z) \in \overline{\mathbf{D}}_{\alpha}$, we consider the stochastic differential equation

$$
d \hat{X}_{t}=-\hat{C}\left(\hat{X}_{t}, \hat{Z}_{t}\right) d t+\hat{\theta}\left(\hat{X}_{t}, \hat{Z}_{t}\right) \sigma\left(d W_{t}+\lambda d t\right)
$$

where we used the previous notation $\hat{Z}_{t}:=z \vee \hat{X}_{t}^{*}, t \geq 0$.

Proposition 6.2 The stochastic differential equation (6.11) has a unique strong solution $(\hat{X}, \hat{Z})$ for any initial condition $(x, z) \in \overline{\mathbf{D}}_{\alpha}$. Moreover,

(i) $(\hat{C}, \hat{\theta})$ satisfies $(3.2)$, and the pair process $\left(C^{*}, \theta^{*}\right):=(\hat{C}, \hat{\theta})\left(\hat{X}_{t}, \hat{Z}_{t}\right) \in \mathcal{A}_{\alpha}^{0}(x, z)$ so that $(\hat{X}, \hat{Z})$ satisfies the strict drawdown constraint $(2.20)$.

(ii) If $\alpha \geq 1 /(1+\gamma)$, the running maximum process is flat, i.e. $\hat{Z}_{t}=z$ for every $t \geq 0$.

This result is based on the following lemmas whose proofs are reported later on.

Lemma 6.2 If $\alpha<1 /(1+\gamma)$, then $\hat{\theta}$ is Lipschitz on $\overline{\mathbf{D}}_{\alpha}$ and $\hat{C}$ is locally Lipschitz on $\mathbf{D}_{\alpha}$.

Lemma 6.3 If $\alpha \geq 1 /(1+\gamma)$, then, for every fixed $z>0, \hat{C}(., z)$ and $\hat{\theta}(., z)$ are locally Lipschitz on $(\alpha z, z)$ and $\hat{\theta}(x, z)=O(\sqrt{z-x})$ near the ray $\left\{(x, z) \in \overline{\mathbf{D}}_{\alpha}, x=z\right\}$.

Lemma 6.4 The functions $\hat{C}$ and $\hat{\theta}$ satisfy

$$
\limsup _{x \searrow \alpha z} \frac{\hat{C}(x, z)}{x-\alpha z}<\infty \quad \text { and } \quad \limsup _{x \searrow \alpha z} \frac{\hat{\theta}(x, z)}{x-\alpha z}<\infty .
$$

Proof of Proposition 6.2 We first observe that, in order to prove item (i) of the proposition, it is sufficient to show that $\left(C^{*}, \theta^{*}\right) \in \mathcal{A}_{\alpha}(x, z)$. Indeed, it follows from lemma 6.4 together with the continuity of the functions $\hat{C}, \hat{\theta}$ that

$$
\int_{0}^{T} \frac{\hat{C}\left(\hat{X}_{t}, \hat{Z}_{t}\right)}{\hat{X}_{t}-\alpha \hat{Z}_{t}} d t+\int_{0}^{T}\left|\frac{\hat{\theta}\left(\hat{X}_{t}, \hat{Z}_{t}\right)}{\hat{X}_{t}-\alpha \hat{Z}_{t}}\right|^{2} d t<\infty \text { a.s. for every } T>0 .
$$


Then the strict drawdown constraint is satisfied by the construction of Section 2.2.

We now prove the remaining claims of the proposition by considering the two alternative situations isolated by lemmas 6.2 and 6.3 .

$\underline{\text { Case } 1}$ Let $\alpha<1 /(1+\gamma)$. We first extend continuously $\hat{C}$ and $\hat{\theta}$ to $\{(x, z): x \leq z\}$ by setting them equal to zero, so that $\hat{\theta}$ and $\hat{C}$ are respectively Lipschitz and locally Lipschitz, see Lemma 6.2.

1. In this step, we show that the stochastic differential equation

$$
d \bar{X}_{t}=\hat{\theta}\left(\bar{X}_{t}, \bar{Z}_{t}\right) \sigma\left(d W_{t}+\lambda d t\right)
$$

has a unique strong solution. To see this, we consider the map $G(t, \mathbf{x}):=\hat{\theta}\left(\mathbf{x}(t), z \vee \mathbf{x}^{*}(t)\right)$ defined on $\mathbb{R}_{+} \times C^{0}\left(\mathbb{R}_{+}\right)$, for fixed $z>0$. Since $\hat{\theta}$ is Lipschitz, We directly estimate, for $t \geq 0$ and $\mathbf{x}, \mathbf{y} \in C^{0}\left(\mathbb{R}_{+}\right)$, that

$$
|G(t, \mathbf{x})-G(t, \mathbf{y})| \leq K\left\{|\mathbf{x}(t)-\mathbf{y}(t)|+\left|z \vee \mathbf{x}^{*}(t)-z \vee \mathbf{y}^{*}(t)\right|\right\} \leq 2 K|\mathbf{x}-\mathbf{y}|_{t}^{*},
$$

where $K>0$ is the Lipschitz constant of $\hat{\theta}$. This proves that $G$ is functional Lipschitz in the sense of Protter [18]. The existence and uniqueness of a strong solution to (6.13) follows from Theorem 7 p 197 in [18].

2. Since $\hat{C}$ is locally Lipschitz on $\mathbf{D}_{\alpha}$ and $\hat{C}(\alpha z, z)=0$ for $z>0$, a similar argument to the above Step 1 shows that local existence and uniqueness hold for the stochastic differential equation (6.11). Recalling that $\hat{C} \geq 0$, it follows from (4.33) that $0 \leq \alpha z \leq \hat{X} \leq \bar{X}$ which shows that there is no explosion of the local solution. Hence $(\hat{X}, \hat{Z})$ is the unique strong global solution to (6.11).

Case 2 Let $\alpha \geq 1 /(1+\gamma)$. The first crucial observation in this case is that $\hat{\theta}(z, z)=0$ by (4.32) and the definition of $\varphi$. Since $\hat{C}$ is non-negative, this shows that any possible solution of the stochastic differential equation (6.11) exhibits a flat component $\hat{Z}_{t}=z$, for every $t \geq 0$. We are then reduced to studying the stochastic differential equation

$$
d \hat{X}_{t}=-\hat{C}\left(\hat{X}_{t}, z\right) d t+\hat{\theta}\left(\hat{X}_{t}, z\right) \sigma\left(d W_{t}+\lambda d t\right)
$$

where $z>0$ is now a fixed parameter. Moreover, it follows from (4.33) that any possible solution of (6.14) must satisfy $\alpha z \leq \hat{X} \leq z$. In view of these bounds, we only need to prove local existence and uniqueness for (6.14). This is guaranteed by Lemma 6.3 and Proposition 2.13 p 291 in [11].

Proof of Lemma 6.2 Notice from lemma 6.1 that $\hat{\theta}$ and $\hat{C}$ are in $C^{1}\left(\mathbf{D}_{\alpha}\right)$. In particular, this implies that $\hat{C}$ is locally Lipschitz on $\mathbf{D}_{\alpha}$. It remains to prove the Lipschitz property of $\hat{\theta}$.

Set $\rho:=\gamma /(1-\alpha(1+\gamma))$ and observe that $0<\rho<\gamma$ under the condition of the lemma. Since $f_{x}$ and $V^{\prime}$ are negative functions, we have

$$
\hat{\theta}_{x}(x, z)=\frac{\lambda}{\sigma}\left(1+\gamma-\frac{\gamma}{\beta} \frac{f_{x}(x, z)}{f(x, z)}\left[V^{\prime} \circ f\right](x, z)\right) \leq \frac{\lambda}{\sigma}(1+\gamma), \quad(x, z) \in \mathbf{D}_{\alpha} .
$$


Notice that, combining the definition of $f$ and (6.3), we get

$$
\begin{aligned}
\frac{\beta}{\gamma} \frac{f(x, z)}{f_{x}(x, z)} & =\left(\frac{\varphi(z)}{f(x, z)}\right)^{1+\gamma} \int_{0}^{\varphi(z)} \frac{V^{\prime}(\xi)}{\xi}\left(\frac{\xi}{\varphi(z)}\right)^{1+\rho} d \xi+\int_{\varphi(z)}^{f(x, z)} \frac{V^{\prime}(\xi)}{\xi}\left(\frac{\xi}{f(x, z)}\right)^{1+\gamma} d \xi \\
& \leq \int_{0}^{f(x, z)} \frac{V^{\prime}(\xi)}{\xi}\left(\frac{\xi}{f(x, z)}\right)^{1+\rho} d \xi, \quad(x, z) \in \mathbf{D}_{\alpha}
\end{aligned}
$$

since $\varphi(z) \leq f(x, z)$ and $\gamma \leq \rho$. Now, since $V^{\prime}$ is a negative increasing function, we deduce

$$
\frac{f(x, z)}{f_{x}(x, z)\left[V^{\prime} \circ f\right](x, z)} \geq \frac{\gamma}{\beta} \int_{0}^{f(x, z)} \frac{1}{\xi}\left(\frac{\xi}{f(x, z)}\right)^{1+\rho} d \xi=\frac{\gamma}{\beta(1+\rho)}>0 .
$$

Combining this inequality with (6.15), we deduce that the function $\theta_{x}$ is bounded on $\mathbf{D}_{\alpha}$. Similarly we compute that, for $(x, z) \in \mathbf{D}_{\alpha}$,

$$
\hat{\theta}_{z}(x, z)=-\frac{\lambda}{\sigma}\left(\alpha(\gamma+1)+\frac{\gamma}{\beta} \frac{f_{z}(x, z)}{f(x, z)}\left[V^{\prime} \circ f\right](x, z)\right) \geq-\frac{\lambda}{\sigma} \alpha(\gamma+1),
$$

since $f_{z}$ and $-V^{\prime}$ are positive functions. Combining (6.3) and (6.4), we compute

$$
\frac{f(x, z)}{f_{z}(x, z)}=-\frac{1}{\alpha}\left(\gamma\left(\frac{\varphi(z)}{f(x, z)}\right)^{1+\gamma}+1\right)^{-1} \frac{f(x, z)}{f_{x}(x, z)} \geq-\frac{1}{\alpha(\gamma+1)} \frac{f(x, z)}{f_{x}(x, z)},
$$

for $(x, z) \in \mathbf{D}_{\alpha}$. We then deduce from (6.17) that $\hat{\theta}_{z}$ is bounded from above and that $\hat{\theta}$ is a Lipschitz function on $\mathbf{D}_{\alpha}$. Since, for any $z>0, \hat{\theta}(\alpha z+, z)=0=\hat{\theta}(\alpha z, z)$, the function $\hat{\theta}$ is in fact Lipschitz on $\overline{\mathbf{D}}_{\alpha}$.

Proof of lemma 6.3 For any fixed $z>0$, we deduce from lemma 6.1 that $\hat{\theta}(., z)$ and $\hat{C}(., z)$ are $C^{1}$ and therefore locally Lipschitz on $(\alpha z, z)$. Observe now that, combining (4.32) with the definition of $f$ in (4.29), we get

$$
\hat{\theta}(x, z)=\frac{2}{\sigma \lambda} \int_{\varphi(z)}^{f(x, z)} \frac{-V^{\prime}(\xi)}{\xi}\left(\frac{\xi}{f(x, z)}\right)^{1+\gamma} d \xi .
$$

Observe also that the definitions (4.28)-(4.29) of $\varphi$ and $f$ lead to

$$
\begin{aligned}
z-x & =(1-\alpha) z-\frac{2}{\lambda^{2}(1+\gamma)} \int_{\varphi(z)}^{\infty} \frac{-V^{\prime}(\xi)}{\xi}\left[\left(\frac{\xi}{f(x, z)}\right)^{1+\gamma} \wedge 1\right] d \xi \\
& =\frac{2}{\lambda^{2}(1+\gamma)} \int_{\varphi(z)}^{f(x, z)} \frac{-V^{\prime}(\xi)}{\xi}\left[1-\left(\frac{\xi}{f(x, z)}\right)^{1+\gamma}\right] d \xi
\end{aligned}
$$

To conclude, we see that, near $x=z$, we have $f(x, z) \sim \varphi(z)$ and therefore

$$
\frac{\theta(x, z)^{2}}{z-x} \sim-\frac{2(1+\gamma) V^{\prime} \circ \varphi(z)}{\sigma^{2}} \frac{\frac{\varphi(z)}{f(x, z)}-1}{\left(\frac{\varphi(z)}{f(x, z)}\right)^{1+\gamma}-1} \sim-\frac{2 V^{\prime} \circ \varphi(z)}{\sigma^{2}} .
$$


Proof of lemma 6.4 Notice that $y:=f(x, z) \sim \infty$ near $x=\alpha z$. Then, by Remark 5.1 together with the expression of $h$ in (4.27), we have, for some constant $K$,

$$
\limsup _{x \searrow \alpha z} \frac{\hat{C}(x, z)}{x-\alpha z}=\limsup _{y / \infty} \frac{-V^{\prime}(y)}{h(y, z)-\alpha z} \cdot \leq \limsup _{y / \infty} K \frac{y^{-1 /(1-p)}}{\max \left(y^{-1 /(1-p)}, y^{-1-\gamma}\right)} \leq K .
$$

The analogue property for $\hat{\theta}$ follows by the same line of argument.

\subsection{Transversality condition}

We finally turn to the proof of the transversality condition (ii-3) of Theorem 3.1.

Proposition 6.3 Given $(x, z) \in \overline{\mathbf{D}}_{\alpha}$, let $(\hat{X}, \hat{Z})$ be the unique strong solution to the stochastic differential equation (6.11). Then, for every sequence of bounded stopping times $\left(\tau_{n}\right)_{n \geq 1}$ with $\tau_{n} \rightarrow \infty$ a.s., we have

$$
\liminf _{n \rightarrow \infty} \mathbb{E}\left[e^{-\beta \tau_{n}} u\left(\hat{X}_{\tau_{n}}, \hat{Z}_{\tau_{n}}\right)\right]=0 .
$$

The proof of this result is organized in the following subsections as follows. Lemma 6.5 shows that the transversality condition holds under a convenient growth condition on the candidate value function. Lemma 6.6 states that the latter growth condition holds in the particular case of a power utility function. By the comparison result of Lemma 6.7, we shall conclude that this growth condition is inherited by any utility function satisfying our asymptotic elasticity condition (6.1).

\subsubsection{Growth condition on the value function}

Lemma 6.5 Let $w: \overline{\mathbf{D}}_{\alpha} \longrightarrow \mathbb{R}$ be such that there exist $K>0$ and $0<\delta<\gamma /(1+\gamma)$ satisfying

$$
|w(x, z)| \leq K\left(1+(x-\alpha z)^{\delta} z^{\frac{\alpha}{1-\alpha}} \delta\right), \quad(x, z) \in \overline{\mathbf{D}}_{\alpha} .
$$

Given $(x, z) \in \overline{\mathbf{D}}_{\alpha}$, let $(C, \theta) \in \mathcal{A}_{\alpha}^{0}(x, z)$ be a consumption-investment strategy satisfying the strict drawdown condition, and $\operatorname{set}(X, Z):=\left(X^{C, \theta}, Z^{C, \theta}\right)$. Assume in addition that

$$
\pi:=\frac{\theta}{X-\alpha Z} \quad \text { is uniformly bounded on } \mathbb{R}_{+} \times \Omega \text {. }
$$

Then, for every sequence of bounded stopping times $\left(\tau_{n}\right)_{n \geq 1}$ with $\tau_{n} \rightarrow \infty$ a.s. we have

$$
\liminf _{n \rightarrow \infty} \mathbb{E}\left[e^{-\beta \tau_{n}} w\left(X_{\tau_{n}}, Z_{\tau_{n}}\right)\right]=0 .
$$

Proof. Denoting by $c:=C /(X-\alpha Z)$, it follows from the representation (2.12) together with the condition of the lemma that

$$
\left|w\left(X_{t}, Z_{t}\right)\right| \leq K\left(1+N_{t} \exp \left\{-\int_{0}^{t} \delta\left(c_{r}-\lambda \sigma \pi_{r}+(1-\delta) \frac{\left(\sigma \pi_{r}\right)^{2}}{2}\right) d r\right\}\right), \quad t>0,
$$


where $N:=\mathcal{E}\left(\int_{0} \sigma \delta \pi_{t} d t\right)$ is the exponential martingale defined by the dynamics

$$
d N_{t}=N_{t} \sigma \delta \pi_{t} d W_{t}
$$

recall that $\pi$ is uniformly bounded. Direct computation shows that

$$
\begin{aligned}
\eta_{s} & :=\beta+\delta\left(c_{s}-\lambda \sigma \pi_{s}+(1-\delta) \frac{\left(\sigma \pi_{s}\right)^{2}}{2}\right) \\
& \geq \frac{\lambda^{2}}{2}\left[\gamma+\delta\left((1-\delta)\left(\frac{\sigma \pi_{s}}{\lambda}-\frac{1}{1-\delta}\right)^{2}-\frac{1}{(1-\delta)}\right)\right] \\
& \geq \frac{\lambda^{2}}{2}\left(\gamma-\frac{\delta}{1-\delta}\right)=: 2 \eta>0
\end{aligned}
$$

since $\delta<\gamma /(1+\gamma)$ by the condition of the lemma. Therefore:

$$
\mathbb{E}\left[e^{-\beta \tau_{n}} w\left(X_{\tau_{n}}, Z_{\tau_{n}}\right)\right] \leq K \mathbb{E}\left[e^{-\beta \tau_{n}}+e^{-2 \eta \tau_{n}} N_{\tau_{n}}\right] .
$$

Furthermore, for any $\varepsilon>0$, it follows from the Hölder inequality:

$$
\begin{aligned}
\mathbb{E}\left[e^{-2 \eta \tau_{n}} N_{\tau_{n}}\right] & \leq\left\|e^{-2 \eta \tau_{n}+\frac{\varepsilon}{2} \int_{0}^{\tau_{n}}\left|\sigma \delta \pi_{t}\right|^{2} d t}\right\|_{\mathbb{L}^{1+\varepsilon^{-1}}} \mathbb{E}\left[\mathcal{E}\left((1+\varepsilon) \sigma \delta \int_{0} \pi_{t} d W_{t}\right)_{\tau_{n}}\right]^{(1+\varepsilon)^{-1}} \\
& =\left\|e^{-2 \eta \tau_{n}+\frac{\varepsilon}{2} \int_{0}^{\tau_{n}}\left|\sigma \delta \pi_{t}\right|^{2} d t}\right\|_{\mathbb{L}^{1+\varepsilon^{-1}}} \\
& \leq\left\|e^{-\left(2 \eta+\frac{\varepsilon}{2} \sigma^{2} \delta^{2}\|\pi\|_{\infty}^{2}\right) \tau_{n}}\right\|_{\mathbb{L}^{1+\varepsilon^{-1}}}
\end{aligned}
$$

Hence for a sufficiently small $\varepsilon>0$, we deduce from (6.19) that

$$
\mathbb{E}\left[e^{-\beta \tau_{n}} w\left(\tilde{X}_{\tau_{n}}, \tilde{Z}_{\tau_{n}}\right)\right] \leq K\left(\mathbb{E}\left[e^{-\beta \tau_{n}}\right]+\left\|e^{-\eta \tau_{n}}\right\|_{\mathbb{L}^{1+\varepsilon^{-1}}}\right)
$$

and the required result follows from the dominated convergence theorem by sending $n$ to infinity.

\subsubsection{Particular case of a power utility function}

In this subsection we specialize the discussion to the case where the utility function and the corresponding dual are given by

$$
U_{p}(x):=\frac{x^{p}}{p}, V_{p}(y)=\frac{y^{-q}}{q} \quad x, y>0 \quad \text { and } \frac{1}{p}-\frac{1}{q}=1,
$$

where $p:=\operatorname{AE}\left(U_{p}\right)$ is a given parameter satisfying (6.1).

The solution of the drawdown problem for this particular type of utility function is much

more tractable. We first observe that the function $\varphi$ defined implicitly by (4.28) rewrites explicitly, for any $z>0$, as

$$
\varphi_{p}(z)=\left[b_{\alpha}(1-\alpha) z\right]^{p-1}, \quad \text { with } \quad b_{\alpha}:=\frac{\beta(1+\gamma)}{\gamma(1-p)^{2}}\left[1 \wedge \frac{\gamma}{(1-\alpha)(1+\gamma)}-p\right]
$$


Remark 6.1 The parameter $b_{0}$ corresponds to the optimal consumption rate in the Merton problem without drawdown constraint. In particular, Assumption 6.1, which is equivalent to $b_{\alpha}>0$, is weaker than the so called merton condition $b_{0}>0$, and reduces to it when $\alpha=0$.

Remark now that the value function $u_{p}^{\alpha}$ given by (2.17) inherits the homogeneity property from the power utility function $U_{p}$, so that $u_{p}^{\alpha}(x, z)=u_{p}^{\alpha}(x / z, 1) z^{p}$, for $(x, z) \in \mathbf{D}_{\alpha}$. Therefore, we naturally expect the function $\hat{C}_{p}$ defined in (4.5) to satisfy $\hat{C}_{p}(x, z)=$ $\hat{C}_{p}(x / z, z) z$, for $(x, z) \in \mathbf{D}_{\alpha}$. Indeed, denoting $f_{p}$ the function defined implicitly by (4.29), direct calculation reveals that the function $(x, z) \mapsto-\left[V_{p}^{\prime} \circ f_{p}\right](x, z) /(x-\alpha z)$ reduces to a function of the single variable $x / z$ :

$f_{p}(x, z)=\left((x-\alpha z) F_{p}^{-1}\left(\frac{x}{z}\right)\right)^{-1+p} \quad$ where $\quad F_{p}(\xi):=\alpha+(1-\alpha) \frac{b_{\alpha}}{\xi}\left(\frac{1-\frac{b_{0}}{\xi}}{1-\frac{b_{0}}{b_{\alpha}}}\right)^{\frac{\lambda^{2}}{2(1-p)^{2}} b_{0}^{-1}}(6.22)$

is invertible as a $C^{1}$ strictly increasing function from $\left[b_{0}^{+}, b_{\alpha}\right]$ to $[\alpha, 1]$. Notice that the case $b_{0}=0$ is also covered by this expression as it can be seen by passing to the limit in $(6.22)$ that:

$$
F_{p}(\xi)=\alpha+(1-\alpha) \frac{b_{\alpha}}{\xi} \exp \left[\frac{1-\alpha}{\alpha \gamma}\left(1-\frac{b_{\alpha}}{\xi}\right)\right] \text { whenever } \quad b_{0}=0 .
$$

For any $(x, z) \in \mathbf{D}_{\alpha}$, we deduce from (6.22) that the candidate value function $u_{p}$ given by (4.30) reduces to

$$
u_{p}(x, z):=\left(\frac{\gamma+1}{\gamma}+\frac{(1-p)^{2}}{\beta p} F_{p}^{-1}\left(\frac{x}{z}\right)\right)\left[F_{p}^{-1}\left(\frac{x}{z}\right)\right]^{p-1}(x-\alpha z)^{p},
$$

and the candidate optimal consumption-investment policy $\left(\hat{C}_{p}, \hat{\theta}_{p}\right)$ given by $(4.31)-(4.32)$ is:

$\hat{C}_{p}(x, z):=(x-\alpha z) F^{-1}\left(\frac{x}{z}\right)$ and $\hat{\theta}_{p}(x, z):=(x-\alpha z)\left(\frac{\lambda}{\sigma}(\gamma+1)-\frac{2}{\sigma \lambda}(1-p) F^{-1}\left(\frac{x}{z}\right)\right)$.

Remark 6.2 The above solution agrees with the candidate solution derived by [19] in the case of possibly positive interest rates. Therefore, Theorem 5.1 confirms that the candidate solution derived by [19] is indeed the solution of the optimal consumption-investment problem.

We now provide some control on the growth of the candidate value function $u_{p}$ which allows to apply lemma 6.5 and leads to the transversality condition in the power utility case.

Lemma 6.6 Let $u_{p}$ be the candidate solution defined in (6.23). Then, for any $z_{0}>0$, there exists $K_{0}>0$ such that

$$
0 \leq u_{p}(x, z) \leq K_{0}(x-\alpha z)^{\delta} z^{\frac{\alpha}{1-\alpha} \delta}, \quad(x, z) \in \overline{\mathbf{D}}_{\alpha}, \quad z \geq z_{0} .
$$

with $\delta:=\left[1-\alpha \wedge \frac{1}{1+\gamma}\right] p$. 
Proof. We first observe that, on the boundary $\overline{\mathbf{D}}_{\alpha} \backslash \mathbf{D}_{\alpha}, u_{p}=0$ and the estimate (6.24) is straightforward. Plugging (6.22) and (6.23) in relation $u_{x}=f$, we easily derive

$\nabla_{x} u_{p}(x, z)=f_{p}(x, z)=u_{p}(x, z)\left(\frac{\gamma+1}{\gamma}(x-\alpha z)+\frac{(1-p)^{2}}{\beta p} F^{-1}\left(\frac{x}{z}\right)(x-\alpha z)\right)^{-1}$,

for $(x, z) \in \mathbf{D}_{\alpha}$, where $\nabla_{x} u_{p}$ denotes the partial derivative of $u_{p}$ with respect to $x$. Since $F^{-1}$ is an increasing function and $F^{-1}(1)=b_{\alpha}$ where $b_{\alpha}$ is defined in (6.21), we get

$$
\frac{\nabla_{x} u_{p}(x, z)}{u_{p}(x, z)} \geq\left[\frac{\gamma}{1+\gamma} \vee(1-\alpha)\right] p(x-\alpha z)^{-1}, \quad(x, z) \in \mathbf{D}_{\alpha} .
$$

Integrating this inequality on the interval $[x, z]$, we obtain, up to the composition with the exponential function

$$
\frac{u_{p}(z, z)}{u_{p}(x, z)} \geq\left(\frac{(1-\alpha) z}{x-\alpha z}\right)^{\delta}, \quad(x, z) \in \mathbf{D}_{\alpha}
$$

By homogeneity, we now observe that $u_{p}(z, z)=u_{p}(1,1) z^{p}$, for any $z>0$, and therefore relation $p-\delta \leq \alpha \delta /(1-\alpha)$ directly leads to (6.24).

\subsubsection{A comparison result}

Lemma 6.7 Let $U_{0}$ and $U_{1}$ be two utility functions satisfying (2.15) and the asymptotic elasticity condition (6.1). Let $u_{0}$ and $u_{1}$ be the corresponding candidate value functions defined by (4.30). Then $U_{0} \leq U_{1}$ implies $u_{0} \leq u_{1}$.

Proof. Since $U_{0} \leq U_{1}$, their Fenchel transforms denoted $V_{0}$ and $V_{1}$ satisfy also $V_{0} \leq V_{1}$. Set $\Delta:=\left(V_{1}-V_{0}\right)$ and $V_{\epsilon}:=V_{0}+\varepsilon \Delta$, for $0 \leq \epsilon \leq 1$, and denote $\varphi_{\epsilon}, f_{\epsilon}$ and $u_{\varepsilon}$ the associated functions defined in section 4.2. Observe first that all these functions are differentiable in $\epsilon$. We shall denote by $\nabla_{\epsilon}$ the gradient operator with respect to $\epsilon$. We intend to prove that $u_{\varepsilon}$ is an increasing function of $\epsilon$ on $[0,1]$, which implies the required result.

For ease of notation, we introduce $\rho:=\gamma /(1-\alpha(1+\gamma)) \in \mathbb{R} \cup\{\infty\}$ and the operator $\Gamma$ defined for $(V, f, \varphi) \in C^{1}\left(\mathbb{R}^{+}, \mathbb{R}^{+}\right) \times \mathbb{R}^{+} \times \mathbb{R}^{+}$by

$\Gamma[V, f, \varphi]:=\int_{f}^{\infty} \frac{-V^{\prime}(\xi)}{\xi} d \xi+\int_{\varphi}^{f} \frac{-V^{\prime}(\xi)}{\xi}\left(\frac{\xi}{f}\right)^{1+\gamma} d \xi+\mathbf{1}_{\left\{\alpha<\frac{1}{1+\gamma}\right\}}\left(\frac{\varphi}{f}\right)^{1+\gamma} \int_{0}^{\varphi} \frac{-V^{\prime}(\xi)}{\xi}\left(\frac{\xi}{\varphi}\right)^{1+\rho} d \xi$

so that, by $(4.29), f_{\varepsilon}$ is defined by

$$
\Gamma\left[V_{\varepsilon}, f_{\varepsilon}, \varphi_{\varepsilon}\right](x, z)=\frac{1+\gamma}{\gamma} \beta(x-\alpha z), \quad(x, z) \in \mathbf{D}_{\alpha},
$$

and by (4.28), the function $\varphi_{\epsilon}$ is implicitly defined by

$$
\Gamma\left[V_{\epsilon}, \varphi_{\epsilon}, \varphi_{\epsilon}\right](z)=\frac{1+\gamma}{\gamma} \beta(1-\alpha) z, \quad z>0 .
$$

Differentiating the latter expression with respect to $\varepsilon$, we see that

$$
\Gamma\left[\Delta, \varphi_{\epsilon}, \varphi_{\epsilon}\right]=\frac{\nabla_{\epsilon} \varphi_{\epsilon}}{\varphi_{\epsilon}}\left(\mathbf{1}_{\left\{\alpha<\frac{1}{1+\gamma}\right\}}(1+\rho) \int_{0}^{\varphi_{\varepsilon}} \frac{-V_{\varepsilon}^{\prime}(\xi)}{\xi}\left(\frac{\xi}{\varphi_{\varepsilon}}\right)^{1+\rho} d \xi-\mathbf{1}_{\left\{\alpha \geq \frac{1}{1+\gamma}\right\}} V_{\varepsilon}^{\prime} \circ \varphi_{\varepsilon}\right) .
$$


We next differentiate (6.27) with respect to $\varepsilon$, and use (6.29), to derive:

$$
\begin{aligned}
(1+\gamma) \frac{\nabla_{\epsilon} f_{\epsilon}}{f_{\varepsilon}}\left(\Gamma\left[V_{\varepsilon}, f_{\varepsilon}, \varphi_{\varepsilon}\right]+\int_{f_{\varepsilon}}^{\infty} \frac{V_{\varepsilon}^{\prime}(\xi)}{\xi} d \xi\right) & =\Gamma\left[\Delta, f_{\varepsilon}, \varphi_{\varepsilon}\right] \\
& -\left[\frac{\alpha \gamma}{1-\alpha} \wedge 1\right]\left(\frac{\varphi_{\varepsilon}}{f_{\varepsilon}}\right)^{1+\gamma} \Gamma\left[\Delta, \varphi_{\varepsilon}, \varphi_{\varepsilon}\right] .
\end{aligned}
$$

We now differentiate (4.30), which in this context is

$$
u_{\varepsilon}=\frac{f_{\varepsilon}}{\beta}\left(\frac{1+\gamma}{\gamma} \beta(x-\alpha z)+\int_{f_{\varepsilon}}^{\infty} \frac{V_{\varepsilon}(\xi)}{\xi^{2}} d \xi\right)
$$

with respect to $\epsilon$, and deduce from integration by part arguments that:

$$
\beta \frac{\nabla_{\varepsilon} u_{\varepsilon}}{f_{\varepsilon}}=\frac{\nabla_{\epsilon} f_{\epsilon}}{f_{\varepsilon}}\left(\frac{1+\gamma}{\gamma} \beta(x-\alpha z)+\int_{f_{\varepsilon}}^{\infty} \frac{V_{\varepsilon}^{\prime}(\xi)}{\xi} d \xi\right)+\int_{f_{\varepsilon}}^{\infty} \frac{\Delta(\xi)}{\xi^{2}} d \xi .
$$

Using (6.27), (6.29) and (6.30), this provides

$$
\begin{aligned}
\beta \frac{\nabla_{\varepsilon} u_{\varepsilon}}{f_{\varepsilon}} & =\frac{1}{1+\gamma}\left[\frac{\alpha \gamma}{1-\alpha} \wedge 1\right]\left(\frac{\varphi_{\varepsilon}}{f_{\varepsilon}}\right)^{1+\gamma} \int_{\varphi_{\varepsilon}}^{\infty} \frac{\Delta(\xi)}{\xi^{2}} d \xi+\frac{\gamma}{1+\gamma} \int_{\varphi_{\varepsilon}}^{f_{\varepsilon}} \frac{\Delta(\xi)}{\xi^{2}}\left(\frac{\xi}{\varphi_{\varepsilon}}\right)^{1+\gamma} d \xi \\
& +\frac{\gamma}{1+\gamma} \int_{f_{\varepsilon}}^{\infty} \frac{\Delta(\xi)}{\xi^{2}} d \xi+1_{\left\{\alpha<\frac{1}{1+\gamma}\right\}} \frac{\rho}{1+\rho}\left(\frac{\varphi_{\varepsilon}}{f_{\varepsilon}}\right)^{1+\gamma} \int_{0}^{\varphi_{\varepsilon}} \frac{\Delta(\xi)}{\xi^{2}}\left(\frac{\xi}{\varphi_{\varepsilon}}\right)^{1+\rho} d \xi
\end{aligned}
$$

for any $\epsilon \in[0,1]$. We now observe that all the above integrals are positive since $\Delta=$ $V_{1}-V_{0} \geq 0$. Combined with $f_{\varepsilon} \geq 0$, this shows that $u_{\varepsilon}$ is non-decreasing in $\varepsilon$ and we deduce $u_{0} \leq u_{1}$.

We can now turn to the

Proof of Proposition 6.3. Since $U$ satisfies (6.1), we recall from (5.2) that $U \leq U_{p}^{0}$ where $U_{p}^{0}=K_{p}\left(1+U_{p}\right)$ and $p:=\mathrm{AE}(\mathrm{U})$ satisfies (6.1). Denoting $u_{p}^{0}$ the candidate value function associated to the utility function $U_{p}^{0}$, we deduce from Lemma 6.7 that $u \leq u_{p}^{0}$. Simple computations show that the marginal utilities $f_{p}^{0}$ and $f_{p}$ associated to the candidate value function $u_{p}^{0}$ and $u_{p}$ are related by $f_{p}^{0}=K_{p} f_{p}$ leading to $u_{p}^{0}=K_{p}\left(1+u_{p}\right)$. Since $u \leq u_{p}^{0}$, we deduce from Lemma 6.6 that

$$
0 \leq u(x, z) \leq K_{0} K_{p}\left(1+z^{\frac{\alpha}{1-\alpha} \delta}(x-\alpha z)^{\delta}\right), \quad(x, z) \in \overline{\mathbf{D}}_{\alpha},
$$

with $\delta:=\left[1-\alpha \wedge \frac{1}{1+\gamma}\right] p<\gamma /(1+\gamma)$ according to (6.1). We finally observe that (4.32) leads to

$$
\hat{\theta}(x, z) \leq \frac{\lambda}{\sigma}(1+\gamma)(x-\alpha z), \quad(x, z) \in \overline{\mathbf{D}}_{\alpha},
$$

so that Lemma 6.5 concludes the proof. 


\section{References}

[1] Barles G., C. Daher and M. Romano (1994). Optimal control of the $\mathbb{L}^{\infty}$-norm of a diffusion process. SIAM Journal on Control and Optimization 32, 612-634.

[2] Ben Tahar I., M. Soner and N. Touzi (2005). Modelling continuous-time financial markets with capital gains taxes. Preprint.

[3] Constantinides G.M. and M.J.P. Magill (1976) Portfolio Selection with Transaction Costs, Journal of Economic Theory 13, 245-263.

[4] Cox J. and C.F. Huang (1989). Optimal consumption and portfolio policies when asset prices follow a diffusion process, Journal of Economic Theory 49, 33-83.

[5] Cvitanić J. and I. Karatzas (1992). Convex duality in constrained portfolio optimization. Annals of Applied Probability 2, 767-818.

[6] Cvitanic J. and I. Karatzas (1995). On portfolio optimization under "drawdown" constraints. IMA volumes in mathematics and its applications.

[7] Davis M.H.A. and A.R. Norman (1990). Portfolio selection with transaction costs. Mathematics of Operations Research 15, 676-713.

[8] El Karoui N. and M. Jeanblanc (1998). Optimization of consumption with labor income. Finance and Stochastics 2, 409-440.

[9] Grossman S.J. and Z. Zhou (1993). Optimal investment strategies for controlling drawdowns. Math. Finance, 3 (3), 241-276.

[10] He H. and H. Pagès (1993). Labor income, borrowing constraints and equilibrium asset prices. Economic Theory 3, 663-696.

[11] Karatzas I. and S.E. Shreve (1991). Brownian Motion and Stochastic Calculus, Springer-Verlag, New York.

[12] Karatzas I. and S.E. Shreve (1998). Methods of Mathematical Finance, SpringerVerlag, New York.

Optimal portfolio and consumption decisions for a "small investor" on a finite horizon. SIAM Journal on Control and Optimization 25, 1557-1586.

[13] Karatzas I., J.P. Lehoczky and S.E. Shreve (1987). Optimal portfolio and consumption decisions for a "small investor" on a finite horizon. SIAM Journal on Control and Optimization 25, 1557-1586.

[14] Klass M.J. and K. Nowicki (2005). The Grossman and Zhou investment strategy is not always optimal. Statistics and Probability Letters 74, 245-252.

[15] Kramkov D. and W. Schachermayer (1999). The condition on the Asymptotic Elasticity of Utility Functions and Optimal Investment in Incomplete Markets. Annals of Applied Probability 9, 904-950. 
[16] Merton R.C. (1969). Lifetime portfolio selection under uncertainty: the continuoustime model. Review of Economic Statistics 51, 247-257.

[17] Merton R.C. (1971). Optimum consumption and portfolio rules in a continuous-time model. Journal of Economic Theory 3, 373-413.

[18] Protter P. (1990). Stochastic integration and differential equations. Springer Verlag, Berlin.

[19] Roche H. (2006). Optimal Consumption and Investment Strategies under Wealth Ratcheting. Preprint.

[20] Schachermayer W. (2001). Optimal Investment in Incomplete Markets when Wealth may Become Negative. Annals of Applied Probability 11, 694-734.

[21] Shreve S.E. and H.M. Soner (1994). Optimal investment and consumption with transaction costs, Annals of Applied Probability 4, 609-692. 